\title{
Existence and Multiplicity Results for Second-Order Nonlinear Differential Equations with Multivalued Boundary Conditions
}

\author{
Droh Arsene Behi, Assohoun Adje \\ Faculty of Mathematics and Computer Science, Felix Houphouet Boigny University, Cocody Abidjan, Ivory Coast \\ Email: arsene.behi@gmail.com, assohoun.adje@univ-fhb.edu.ci
}

How to cite this paper: Behi, D.A. and Adje, A. (2019) Existence and Multiplicity Results for Second-Order Nonlinear Differential Equations with Multivalued Boundary Conditions. Journal of Applied Mathematics and Physics, 7, 1340-1368. https://doi.org/10.4236/jamp.2019.76091

Received: May 16, 2019

Accepted: June 27, 2019

Published: June 30, 2019

\section{Copyright $\odot 2019$ by author(s) and} Scientific Research Publishing Inc. This work is licensed under the Creative Commons Attribution International License (CC BY 4.0).

http://creativecommons.org/licenses/by/4.0/

\begin{abstract}
In this paper, we consider the following second-order nonlinear differential equations' problem: $-\left(\Phi\left(u^{\prime}(t)\right)\right)^{\prime}=f\left(t, u(t), u^{\prime}(t)\right)+\Xi(u(t))$ a.e on $\Omega=[0, T]$ with a discontinuous perturbation and multivalued boundary conditions. By combining lower and upper solutions method, theory of monotone operators and theory of topological degree, we show the existence of solutions of the investigated problem in two cases. At first, $\alpha$ and $\beta$ are assumed respectively an ordered pair of lower and upper solutions of the problem, secondly $\alpha$ and $\beta$ are assumed respectively non ordered pair of lower and upper solutions of the problem. Moreover, we show multiplicity results when the problem admits a pair of lower and strict lower solutions and a pair of upper and strict upper solutions. We also show that our method of proof stays true for a periodic problem.
\end{abstract}

\section{Keywords}

$\Phi$-Laplacian, Lower and Upper Solutions, Maximal Monotone Maps, Bernstein-Nagumo-Wintner Growth Condition, Leray-Schauder Topological Degree

\section{Introduction}

This paper is devoted to the study of the following problem:

$$
\left\{\begin{array}{l}
-\left(\Phi\left(u^{\prime}(t)\right)\right)^{\prime}=f\left(t, u(t), u^{\prime}(t)\right)+\Xi(u(t)) \text { a.e on } \Omega=[0, T] \\
u^{\prime}(0) \in B_{1}(u(0)),-u^{\prime}(T) \in B_{2}(u(T))
\end{array}\right.
$$

where $B_{1}$ and $B_{2}$ are maximal monotone graphs in $\mathbb{R}^{2}$ and are some mul- 
tifunctions which describe the boundary conditions, $f: \Omega \times \mathbb{R}^{2} \rightarrow \mathbb{R}$ is a Caratheodory function and $\Phi$ is an increasing homeomorphism such that $\Phi(0)=0$.

The tool of investigation for this problem is lower and upper solution's method. This method provides a precious tool to get existence results for first and second order initial and boundary value problems. The method allows to generate monotone iterative techniques which provide constructive methods (amenable numerical treatment), to obtain solutions. The method was first introduced by Perron [1]. Later Nagumo [2] used upper and lower solutions to study second-order differential equations with Dirichlet boundary conditions. Since then many authors have used that method primarily in the context of single-valued differential equations with linear boundary conditions (Dirichlet, Neumann, Sturm-Liouville or periodic). Very recently, in 2017, combining lower and upper solutions method and theory of topological degree, Goli-Adjé [3] established existence and multiplicity results for the considered problem under Neumann-Steklov boundary value conditions. Shortly before, in 2013, Khattabi-Frigon-Ayyadi [4], by lower and upper solutions method and the fixed point index theory, obtained existence and multiplicity results for the problem under various boundary value conditions (Dirichlet, periodic or Neumann). Other authors have investigated the second-order differential equation with multivalued boundary conditions driven by maximal monotone operators, in this direction, see [5] [6] [7] [8] [9] and references therein. In [6] [7], the problems unify classical problems of Dirichlet, periodic and Neumann and in [5] [8] [9] the problems unify classical problems of Dirichlet, Neumann and Sturm-Louiville. To our knowledge, the lower and upper solutions method for differential inclusions formulation of problems of type (1) was initiated by Bader-Papageorgiou [5] in 2002. Soon after, in 2006, Staicub and Papageorgiou [9] extended the study of that problem to gradient systems with a discontinuous nonlinearity. In 2007, Kyritsi and Papageorgiou [8], in their book (see [8] the problem (5.111), p. 390) investigated the following single-valued version of the problem in Staicub-Papageorgiou [9]:

$$
\left\{\begin{array}{l}
-\left(a\left(u^{\prime}(t)\right) \Phi_{p}\left(u^{\prime}(t)\right)\right)=f\left(t, u(t), u^{\prime}(t)\right)+\Xi(u(t)) \text { a.e on } \Omega=[0, T] \\
u^{\prime}(0) \in B_{1}(u(0)),-u^{\prime}(T) \in B_{2}(u(T))
\end{array}\right.
$$

where $B_{1}, B_{2}, \Xi$ and $f$ are defined as in problem (1) and for all $z \in \mathbb{R}$, $a(z)=1$. So, in [5] [8] [9], the authors deal with the homogeneous operator differential $p$-laplacian $\Phi_{p}(z)=|z|^{p-2} z$ for all $z \in \mathbb{R}$. But they do not establish multiplicity results.

The goal of this paper is to extend the work of Kyritsi-Papageorgiou [8] to a large class of problems. Indeed, we deal with a non-homogeneous operator $\Phi$ -laplacian which contains, for example, some versions of $\Phi$-Laplacian operators like the case when, for all $z \in \mathbb{R}, \Phi(z)=a(z) \Phi_{p}(z)$ with $\left.a: \mathbb{R} \rightarrow\right] 0,+\infty[$ is a continuous map. Moreover, to obtain multiplicity results, we combine lower 
and upper method used in [5] [8] [9] and the one of Goli-Adjé [3]. So, our aim in this paper is to study existence and multiplicity results concerning solutions of problem (1).

After introducing notations, preliminary results and auxiliary results in Section 2 and Section 3, in Section 4, $\alpha$ and $\beta$ are assumed respectively an ordered pair of lower and upper solutions of the problem. By combining lower and upper solutions method and theory of topological degree we obtain existence results.

In Section 5, $\alpha$ and $\beta$ are assumed respectively non-ordered pair of lower and upper solutions of the problem. Also, by combining lower and upper solutions method and topological degree theory, we obtain existence results.

In Section 6, using the aforementioned method in Section 4 and 5, we show multiplicity results at first when the problem admits a pair of lower and strict lower solutions and a pair of upper and strict upper solutions, secondly when the problem admits two lower solutions and a strict upper solution or when the problem admits a strict lower solution and two upper solutions.

In Section 7, we give an example of application and we show also, as in [8], that our method stays true for the periodic problem.

In Section 8, we give a conclusion.

\section{Preliminaries}

In this section, we introduce our terminology and notations. We also recall some basic definitions and facts from multivalued analysis that we will need in the sequel. Our main sources are the books of Hu-Papageorgiou [10] and Zeidler [11].

The Sobolev spaces $W^{1, p}(\Omega)$ and $L^{p}(\Omega)$ are respectively equipped with the norms:

$$
\|y\|=\left(\int_{0}^{T}|y(t)|^{p}+\left|y^{\prime}(t)\right|^{p}\right)^{\frac{1}{p}} \text { and }\|u\|_{p}=\left(\int_{0}^{T}|u(t)|^{p} \mathrm{~d} t\right)^{\frac{1}{p}} .
$$

The space of continuous function $C(\Omega)$ is endowed with the norm:

$$
\|u\|_{\infty}=\max \{|u(t)|: t \in \Omega\} \text {. }
$$

We denote: $\rightarrow$ : the weak convergence; $\rightarrow$ : the strong convergence; $|x|$ : absolute value of $x ; s^{+}=\max \{s, 0\} ; \mathrm{R}(A)$ : image of operator $A ; d_{L S}$ : Leray-Schauder's degree; $\mathbb{R}_{+} \backslash\{0\}=\mathbb{R}_{+}^{*} ; P\left(X^{*}\right)$ : the family of subsets of space $X^{*}$.

Let $X$ be a reflexive Banach space and $X^{*}$ the topological dual of $X$. A map $A: D(A) \subseteq X \rightarrow P\left(X^{*}\right)$ is said to be monotone, if for all $x, y \in D(A)$ and for all $x^{*} \in A(x), y^{*} \in A(y)$, we have $\left\langle x^{*}-y^{*}, x-y\right\rangle \geq 0$ by $\langle$.$\rangle we denote the$ duality brackets for the pair $\left(X, X^{*}\right)$. If additionally, the fact that $\left\langle x^{*}-y^{*}, x-y\right\rangle=0$ implies that $x=y$, then we say that $A$ is strictly monotone. The map $A$ is said to be maximal monotone, if it is monotone and for all $x \in D(A), x^{*} \in A(x)$, the fact that $\left\langle x^{*}-y^{*}, x-y\right\rangle \geq 0$ implies that $y \in D(A)$ and $y^{*} \in A(y)$. It is clear from this definition that $A$ is maximal monotone if 
and only if its graph $\operatorname{Gr} A=\left\{\left(x, x^{*}\right) \in X \times X^{*}: x^{*} \in A(x)\right\}$ is maximal with respect to inclusion among the graphs of monotone maps. If $A$ is maximal monotone, for any $x \in D(A)$, the set $A(x)$ is non-empty, closed and convex. Moreover, GrA is demi-closed, i.e., if $\left(x_{n}, x_{n}^{*}\right) \in \mathrm{Gr} A, n \geq 1$, either $x_{n} \rightarrow x$ in $X$ and $x_{n}^{*} \rightarrow x^{*}$ in $X^{*}$, or $x_{n} \rightarrow x$ in $X$ and $x_{n}^{*} \rightarrow x^{*}$ in $X^{*}$, then $\left(x, x^{*}\right) \in \mathrm{Gr}$. If $A: X \rightarrow X^{*}$ is everywhere defined and single-valued, we say that $A$ is demi-continuous, if for every sequence $\left(x_{n}\right)_{n \geq 1}$ such that $x_{n} \rightarrow x$ in $X$, we have that $A\left(x_{n}\right) \rightarrow A(x)$ in $X^{*}$. If map $A: X \supseteq D(A) \rightarrow X^{*}$ is monotone and demi-continuous, then it is also maximal monotone. A map $A: X \supseteq D(A) \rightarrow P\left(X^{*}\right)$ is said to be coercive, if $D(A) \subseteq X$ is bounded or if $D(A)$ is unbounded and we have that

$$
\frac{\inf \left\{\left\langle x^{*}, x\right\rangle_{X}: x^{*} \in A(x)\right\}}{\|x\|_{X}} \rightarrow+\infty \text { as }\|x\|_{X} \rightarrow+\infty, x \in D(A) .
$$

A maximal monotone and coercive map is surjective. Let $Y, Z$ be Banach spaces and $L: Y \rightarrow Z$. We say:

a) $L$ is "completely continuous", if $y_{n}-y$ in $Y$ implies $L\left(y_{n}\right) \rightarrow L(y)$ in $Z$ and

b) $L$ is "compact", if it is continuous and maps bounded sets into relatively compact sets.

In general, these two notions are distinct. However, if $Y$ is reflexive, then complete continuity implies compactness. Moreover, if $Y$ is reflexive and $L$ is linear, then the two notions are equivalent.

\section{Auxiliary Results}

Let $p, q \in \mathbb{N}^{*}$ such that $\frac{1}{p}+\frac{1}{q}=1, p \geq 2$. First, let us define what we mean by solution of problem (1).

Definition 1. A function $u \in C^{1}(\Omega)$ such that $\Phi\left(u^{\prime}().\right) \in W^{1, q}((0, T))$ is said to be a solution of the problem (1), if it verifies (1).

Next, we introduce the notions of upper and lower solutions of problem (1).

\section{Definition 2.}

a) A function $\beta \in C^{1}(\Omega)$ such that $\Phi\left(\beta^{\prime}().\right) \in W^{1, q}((0, T))$ is said to be an upper solution of the problem (1), if.

$$
\left\{\begin{array}{l}
-\left(\Phi\left(\beta^{\prime}(t)\right)\right) \geq f\left(t, \beta(t), \beta^{\prime}(t)\right)+\Xi(\beta(t)) \text { a.e on } \Omega=[0, T] \\
\beta^{\prime}(0) \in B_{1}(\beta(0))-\mathbb{R}_{+},-\beta^{\prime}(T) \in B_{2}(\beta(T))-\mathbb{R}_{+} .
\end{array}\right.
$$

b) A function $\alpha \in C^{1}(\Omega)$ such that $\Phi\left(\alpha^{\prime}().\right) \in W^{1, q}((0, T))$ is said to be a lower solution of problem (1), if.

$$
\left\{\begin{array}{l}
-\left(\Phi\left(\alpha^{\prime}(t)\right)\right) \leq f\left(t, \alpha(t), \alpha^{\prime}(t)\right)+\Xi(\alpha(t)) \text { a.e on } \Omega=[0, T] \\
\alpha^{\prime}(0) \in B_{1}(\alpha(0))+\mathbb{R}_{+},-\alpha^{\prime}(T) \in B_{2}(\alpha(T))+\mathbb{R}_{+} .
\end{array}\right.
$$

Now, let us specify what we mean by strict lower and strict upper solutions of 
problem (1).

Definition 3. A lower solution $\alpha$ of (1) is said to be strict if all solution $u$ of (1) with $u(t) \geq \alpha(t), \forall t \in[0, T]$ is such that $u(t)>\alpha(t), \forall t \in[0, T]$.

Definition 4. An upper solution $\beta$ of $(1)$ is said to be strict if all solution $u$ of (1) with $u(t) \leq \beta(t), \forall t \in[0, T]$ is such that $u(t)<\beta(t), \forall t \in[0, T]$.

Proposition 5. Let $\alpha$ be a lower solution of (1) such that:

i) For all $\left.t_{0} \in\right] 0, T\left[\right.$, there exists $\varepsilon_{0}>0$ and $\Omega_{0}$ is an open interval such that $t_{0} \in \Omega_{0}$ and:

$$
\begin{gathered}
-\left(\Phi\left(\alpha^{\prime}(t)\right)\right) \leq f(t, x, y)+\Xi(x) \quad \text { a.e } t \in \Omega_{0}, \\
\text { for all }(x, y) \in\left[\alpha(t), \alpha(t)+\varepsilon_{0}\right] \times\left[\alpha^{\prime}(t)-\varepsilon_{0}, \alpha^{\prime}(t)+\varepsilon_{0}\right] ;
\end{gathered}
$$

ii) $\alpha^{\prime}(0) \in B_{1}(\alpha(0))+\mathbb{R}_{+}^{*}$;

iii) $-\alpha^{\prime}(T) \in B_{2}(\alpha(T))+\mathbb{R}_{+}^{*}$;

then $\alpha$ is a strict lower solution of (1).

Proof. Let $u$ be a solution of problem (1) such that $\alpha(t) \leq u(t)$ for all $t \in \Omega$.

Let us assume that $u$ is not strict, then there exists $\bar{t} \in[0, T]$ such that $\alpha(\bar{t})=u(\bar{t})$. Whence

$$
A=\{t \in[0, T]: \alpha(t)=u(t)\} \neq \varnothing
$$

$A$ is closed and bounded. Let $t_{0}=\min A$. Then

$$
\min _{[0, T]}[u(t)-\alpha(t)]=\alpha\left(t_{0}\right)-u\left(t_{0}\right)
$$

a) If $\left.t_{0} \in\right] 0, T\left[\right.$, then $u^{\prime}\left(t_{0}\right)-\alpha^{\prime}\left(t_{0}\right)=0$ and there exist $\Omega_{0}$ and $\epsilon_{0}>0$ according to (i). We can choose $t_{1} \in \Omega_{0}$ such that $t_{1}<t_{0}, u^{\prime}\left(t_{1}\right)<\alpha^{\prime}\left(t_{1}\right)$, and

$$
\left.\forall t \in\left[t_{1}, t_{0}\right],\left(u(t), u^{\prime}(t)\right) \in\right] \alpha(t), \alpha(t)+\epsilon_{0}[\times] \alpha^{\prime}(t)-\epsilon_{0}, \alpha^{\prime}(t)+\epsilon_{0}[.
$$

Therefore, for almost $t \in\left[t_{1}, t_{0}\right]$,

$$
-\left(\Phi\left(\alpha^{\prime}(t)\right)\right)^{\prime}-f\left(t, u(t), u^{\prime}(t)\right)-\Xi(u(t)) \leq 0 .
$$

Since $\Phi$ is an increasing homeomorphism, we have

$$
\Phi\left(u^{\prime}\left(t_{0}\right)\right)-\Phi\left(\alpha^{\prime}\left(t_{0}\right)\right)=0
$$

and

$$
\Phi\left(u^{\prime}\left(t_{1}\right)\right)<\Phi\left(\alpha^{\prime}\left(t_{1}\right)\right)
$$

Also we have:

$$
\begin{aligned}
& \Phi\left(u^{\prime}\left(t_{1}\right)\right)-\Phi\left(\alpha^{\prime}\left(t_{1}\right)\right) \\
& =-\int_{t_{1}}^{t_{0}}\left(\Phi\left(u^{\prime}(s)\right)\right)^{\prime}-\left(\Phi\left(\alpha^{\prime}(s)\right)\right)^{\prime} \mathrm{d} s \\
& =-\int_{t_{1}}^{t_{0}}-f\left(s, u(s), u^{\prime}(s)\right)-\Xi(u(s))-\left(\Phi\left(\alpha^{\prime}(s)\right)\right)^{\prime} \mathrm{d} s \geq 0
\end{aligned}
$$

which contradicts (2).

b) We suppose that $t_{0}=0$, then $\alpha^{\prime}(0)=u^{\prime}(0)$ and it follows that: 


$$
\Phi\left(u^{\prime}(0)\right)-\Phi\left(\alpha^{\prime}(0)\right) \geq 0 .
$$

Since $\alpha^{\prime}(0) \in B_{1}(\alpha(0))+e_{0}, e_{0}>0$, because of the monotonicity of $B_{1}$, if $\alpha(0) \geq u(0)$, we have:

$$
\begin{gathered}
\alpha^{\prime}(0)>u^{\prime}(0) \text {. Then, } \Phi\left(\alpha^{\prime}(0)\right)>\Phi\left(u^{\prime}(0)\right) \\
\text { So } \Phi\left(u^{\prime}(0)\right)-\Phi\left(\alpha^{\prime}(0)\right)<0
\end{gathered}
$$

which contradicts (3).

c) We suppose that $t_{0}=T$, then $u^{\prime}(T)=\alpha^{\prime}(T)$. It follows that:

$$
\Phi\left(u^{\prime}(T)\right)-\Phi\left(\alpha^{\prime}(T)\right) \leq 0 .
$$

Since $-\alpha^{\prime}(T) \in B_{2}(\alpha(T))+e_{0}, e_{0}>0$, because of the monotonicity of $B_{1}$, if $\alpha(T) \geq u(T)$, we have:

$$
\begin{gathered}
\alpha^{\prime}(T)<u^{\prime}(T) \text {. Then, } \Phi\left(\alpha^{\prime}(T)\right)<\Phi\left(u^{\prime}(T)\right) . \\
\text { So } \Phi\left(u^{\prime}(T)\right)-\Phi\left(\alpha^{\prime}(T)\right)>0
\end{gathered}
$$

which contradicts 4 . Then, $t_{0}$ does not exist. So, $A=\varnothing$.

Proposition 6. Let $\beta$ be an upper solution of (1) such that.

i) For all $\left.t_{0} \in\right] 0, T\left[\right.$, there exist $\varepsilon_{0}>0$ and $\Omega_{0}$ is an open interval such that $t_{0} \in \Omega_{0}$ and:

$$
\begin{gathered}
-\left(\Phi\left(\beta^{\prime}(t)\right)\right) \geq f(t, x, y)+\Xi(x) \text { a.e } t \in \Omega_{0}, \\
\text { for all }(x, y) \in\left[\beta(t)-\varepsilon_{0}, \beta(t)\right] \times\left[\beta^{\prime}(t)-\varepsilon_{0}, \beta^{\prime}(t)+\varepsilon_{0}\right] ;
\end{gathered}
$$

ii) $\beta^{\prime}(0) \in B_{1}(\beta(0))-\mathbb{R}_{+}^{*}$;

iii) $-\beta^{\prime}(T) \in B_{2}(\beta(T))-\mathbb{R}_{+}^{*}$;

then $\beta$ is a strict upper solution of (1).

Proof. Let $u$ be a solution of problem (1) such that $u(t) \leq \beta(t)$ for all $t \in \Omega$.

Let us assume that $u$ is not strict, then there exists $\bar{t} \in[0, T]$ such that $\beta(\bar{t})=u(\bar{t})$. Whence

$$
E=\{t \in[0, T]: u(t)=\beta(t)\} \neq \varnothing
$$

$E$ is closed and bounded. Let $t_{0}=\min E$. Then

$$
u^{\prime}\left(t_{0}\right)-\beta^{\prime}\left(t_{0}\right)=0
$$

a) If $\left.t_{0} \in\right] 0, T\left[\right.$ there exist $\Omega_{0}$ and $\epsilon_{0}>0$ according to (i). We can choose $t_{1} \in \Omega_{0}$ such that $t_{1}<t_{0}, u^{\prime}\left(t_{1}\right)>\beta^{\prime}\left(t_{1}\right)$ and

$$
\left.\forall t \in\left[t_{1}, t_{0}\right],\left(u(t), u^{\prime}(t)\right) \in\right] \beta(t)-\epsilon_{0}, \beta(t)[\times] \beta^{\prime}(t)-\epsilon_{0}, \beta^{\prime}(t)+\epsilon_{0}[.
$$

Therefore, for almost $t \in\left[t_{1}, t_{0}\right]$,

$$
-\left(\Phi\left(\beta^{\prime}(t)\right)\right)^{\prime}-f\left(t, u(t), u^{\prime}(t)\right)-\Xi(u(t)) \geq 0 .
$$

Since $\Phi$ is an increasing homeomorphism, we have

$$
\Phi\left(\beta^{\prime}\left(t_{0}\right)\right)-\Phi\left(u^{\prime}\left(t_{0}\right)\right)=0
$$

and 


$$
\Phi\left(u^{\prime}\left(t_{1}\right)\right)>\Phi\left(\beta^{\prime}\left(t_{1}\right)\right)
$$

Also we have:

$$
\begin{aligned}
& \Phi\left(u^{\prime}\left(t_{1}\right)\right)-\Phi\left(\beta^{\prime}\left(t_{1}\right)\right) \\
& =-\int_{t_{1}}^{t_{0}}\left(\Phi\left(u^{\prime}(s)\right)\right)^{\prime}-\left(\Phi\left(\beta^{\prime}(s)\right)\right)^{\prime} \mathrm{d} s \\
& =-\int_{t_{1}}^{t_{0}}\left(-\Phi\left(\beta^{\prime}(s)\right)\right)^{\prime}-f\left(s, u(s), u^{\prime}(s)\right)-\Xi(u(s)) \mathrm{d} s \leq 0
\end{aligned}
$$

which contradicts (5).

b) We suppose that $t_{0}=0$, then $\beta^{\prime}(0)=u^{\prime}(0)$ and it follows that:

$$
\Phi\left(u^{\prime}(0)\right)-\Phi\left(\beta^{\prime}(0)\right) \leq 0 .
$$

Since $\beta^{\prime}(0) \in B_{1}(\beta(0))-e_{0}, e_{0}>0$, because of the monotonicity of $B_{1}$, if $\beta(0) \leq u(0)$, we have:

$$
\begin{gathered}
\beta^{\prime}(0)<u^{\prime}(0) \text {. Then } \Phi\left(\beta^{\prime}(0)\right)<\Phi\left(u^{\prime}(0)\right) \\
\text { So } \Phi\left(u^{\prime}(0)\right)-\Phi\left(\beta^{\prime}(0)\right)>0
\end{gathered}
$$

which contradicts (6).

c) We suppose that $t_{0}=T$, then $u^{\prime}(T)=\beta^{\prime}(T)$. It follows that:

$$
\Phi\left(u^{\prime}(T)\right)-\Phi\left(\beta^{\prime}(T)\right) \geq 0 \text {. }
$$

Since $-\beta^{\prime}(T) \in B_{2}(\beta(T))-e_{0}, e_{0}>0$, because of the monotonicity of $B_{1}$, if $\beta(T) \leq u(T)$, we have:

$$
\begin{gathered}
\beta^{\prime}(T)>u^{\prime}(T) \text {. Then } \Phi\left(\beta^{\prime}(T)\right)>\Phi\left(u^{\prime}(T)\right) . \\
\text { So } \Phi\left(u^{\prime}(T)\right)-\Phi\left(\beta^{\prime}(T)\right)<0
\end{gathered}
$$

which contradicts (7). Then, $t_{0}$ does not exist. So, $E=\varnothing$.

Remark 7. In general, for a given problem, there is not a methodology (single valued and multivalued alike) which allows generating a lower and upper solutions. But, one should try simple functions such as constants, linear, quadratic, exponentials, eigenfunctions of simple operator, etc.

We make the following hypotheses on the data of (1):

$\left(H_{0}\right)$ : There exists a lower solution $\alpha \in C^{1}(\Omega)$ and an upper solution $\beta \in C^{1}(\Omega)$.

$\left(H_{\Phi}\right) \Phi: \mathbb{R} \rightarrow \mathbb{R}$ is an increasing continuous map such that:

a) $\Phi(0)=0$;

b) there exists $d_{1}>0$ such that: $\Phi(x) x \geq d_{1}|x|^{p}$ for all $x \in \mathbb{R}$;

c) there exist $d_{2}, d_{3}>0$ such that for a.e $t \in \Omega$ and for all $x \in \mathbb{R}$ :

$$
|\Phi(x)| \leq d_{2}+d_{3}|x|^{p-1} \text {. }
$$

Remark 8. Suppose that $\Phi(z)=\Phi_{p}(z)=|z|^{p-2} z, p \geq 2$. Then this function satisfies hypothesis $\left(H_{\Phi}\right)$. This function corresponds to the one-dimensional operator p-Laplacian. Another interesting case which satisfies hypothesis $\left(H_{\Phi}\right)$ is when $\Phi$ is defined by $\Phi(z)=\varphi(|z|)|z|^{p-2} z$ with $\varphi: \mathbb{R}_{+} \rightarrow \mathbb{R}_{+}$continuous, 
$\varphi(y) \geq k>0$ for all $y \geq 0$ and $y \mapsto \varphi(y) y^{p-1}$ is strictly increasing on $\mathbb{R}_{+}$ and $\varphi(y) y^{p-1} \leq d_{2}+d_{3} y^{p-1}$. For example, we can have

$$
\varphi(y)=\frac{\sqrt{1+\left(1+y^{p-1}\right)^{2}}}{1+y^{p-1}} \text { or } \varphi(y)=1+\frac{1}{1+y^{p-1}} .
$$

It is well-known that under the monotonicity condition and the hypotheses (a) and (b), $\Phi$ is an increasing homeomorphism from $\mathbb{R}$ onto $\mathbb{R}$. And $\Phi^{-1}$ is strictly monotone and $\left|\Phi^{-1}(y)\right| \rightarrow+\infty$ as $|y| \rightarrow+\infty$ (See Deimling [12] chap. 3). Our operator $\Phi$ is a slightly more restrictive version of the scalar case of the operator used by Sophia Kirytsi-N. Matzakos [13] and Manasevich-Mawhin [14] where growth condition $|\Phi(x)| \leq d_{2}+d_{3}|x|^{p-1}$ is not assumed. Nevertheless, it incorporates the operator p-laplacian and many other classes of operators.

$\left(H_{f}\right) \quad f: \Omega \times \mathbb{R} \times \mathbb{R} \rightarrow \mathbb{R}$ is a fonction such that:

i) for all $x, y \in \mathbb{R}, t \mapsto f(t, x, y)$ is measurable;

ii) for a.e $t \in \Omega,(x, y) \mapsto f(t, x, y)$ is continuous;

iii) for a.e $t \in \Omega, x \in[\alpha(t), \beta(t)]$ and all $y \in \mathbb{R}$, we have:

$$
|f(t, x, y)|<\eta(|\Phi(y)|)(\psi(t)+c|y|)
$$

where $\psi \in L^{1}(\Omega)_{+}, c>0$ and $\eta: \mathbb{R}_{+} \rightarrow \mathbb{R}_{+} \backslash\{0\}$ a Borel measurable non-decreasing functions such that:

$$
\begin{aligned}
\int_{\Phi(\zeta)}^{+\infty} \frac{\mathrm{d} s}{\eta(s)}> & \|\psi\|_{1}+c\left(\max _{\Omega} \beta-\min _{\Omega} \alpha\right) \\
& +\frac{T}{\eta(\zeta)} \sup \left\{|\Xi(z)|:|z| \leq \max \left\{\|\alpha\|_{\infty},\|\beta\|_{\infty}\right\}\right\}
\end{aligned}
$$

with $\zeta=\frac{\max \{|\alpha(T)-\beta(0)|,|\alpha(0)-\beta(T)|\}}{T}$;

iv) for every $r>0$, there exists $\gamma_{r} \in L^{q}(\Omega)$ such that for a.e $t \in \Omega$ and for all $x, y \in \mathbb{R}$ with $|x|,|y| \leq r$ we have: $|f(t, x, y)| \leq \gamma_{r}(t)$.

Remark 9. Hypothesis $\left(H_{f}\right)$ (iii) is known as a Bernstein-Nagumo-Wintner growth condition and produces a uniform a priori bound of the derivatives of the solutions of problem (1). And the hypotheses $\left(H_{f}\right)$ (i), (ii) and (iv) are well known as $L^{p}$-Carathéodory conditions.

$\left(H_{B}\right): B_{1}$ and $B_{2}: \mathbb{R} \rightarrow P(\mathbb{R})$ are maximal monotone maps such that $0 \in B_{1}(0) \cap B_{2}(0)$.

Remark 10. There exist functions $E_{1}, E_{2}: \mathbb{R} \rightarrow \mathbb{R} \cup\{+\infty\}$ proper, convex and lower semi-continuous which are not identically equal to $+\infty$ such that $B_{1}=\partial E_{1}, B_{2}=\partial E_{2}$. More exactly, there exists some increasing positives functions $p_{1}$ and $p_{2}$ such that $p_{i}(s)=\operatorname{Proj}\left(0 ; B_{i}(s)\right.$ ) (the minimum absolute value element in the closed, convex set $\left.B_{i}(s)\right)$. Then $E_{i}(s)=\int_{0}^{s} p_{i}(t) \mathrm{d} t, i=1,2$. We have $B_{i}(s)=\left[p_{i}(s-) ; p_{i}(s+)\right]$ for all $s \in \mathbb{R}$, where

$$
p_{i}(s-)=\lim _{\varepsilon \rightarrow 0^{+}} p_{i}(s-\varepsilon) \text { and } p_{i}(s+)=\lim _{\varepsilon \rightarrow 0^{+}} p_{i}(s+\varepsilon), \quad i=1,2 \text {. }
$$

$\left(H_{\Xi}\right): \Xi: \mathbb{R} \rightarrow \mathbb{R}$ is a function that maps bounded sets to bounded sets and 
there exists $M>0$ such that $x \rightarrow \Xi(x)+M x$ is increasing.

Remark 11. We emphasize that $\Xi$ need not be continuous.

Lemma 1. If $u \in C^{1}(\Omega)$ and hypotheses $\left(H_{\Phi}\right)$ and $\left(H_{f}\right)$ (iii) hold,

$$
-\left(\Phi\left(u^{\prime}(t)\right)\right)^{\prime}=f\left(t, u(t), u^{\prime}(t)\right)+\Xi(u(t)) \text { a.e on } \Omega=[0, T]
$$

and if

$$
\alpha(t) \leq u(t) \leq \beta(t) \text { for all } t \in \Omega
$$

then, there exists $M_{1}>0$ (depending only on $\alpha, \beta, \eta, \psi, \Xi, c$ ) such that: $\left|u^{\prime}(t)\right| \leq M_{1}$ for all $t \in \Omega$.

Proof. Set $\mu=\left(\frac{1}{\eta(\zeta)}\right) \sup \left\{|\Xi(z)|:|z| \leq \max \left\{\|\alpha\|_{\infty},\|\beta\|_{\infty}\right\}\right\} \quad$ (See hypothesis $\left.H_{\Xi}\right)$. By hypothesis $\left(H_{f}\right)$ (iii), we can find $M_{1}>\zeta$ such that

$$
\int_{\Phi(\zeta)}^{\Phi\left(M_{1}\right)} \frac{\mathrm{d} s}{\eta(s)}>\|\psi\|_{1}+c\left(\max _{\Omega} \beta-\min _{\Omega} \alpha\right)+\mu T
$$

We claim that $\left|u^{\prime}(t)\right| \leq M_{1}$ for all $t \in \Omega$. Let's suppose that it is not the case. Then, we can find $t_{1} \in \Omega$ such that

$$
\left|u^{\prime}\left(t_{1}\right)\right|>M_{1} .
$$

By the mean value theorem, there exists $t_{2} \in(0, T)$ such that $u(T)-u(0)=u^{\prime}\left(t_{2}\right) T$. Without any loss of generality, we assume that $t_{2} \leq t_{1}$. We obtain:

$\left|u^{\prime}\left(t_{2}\right)\right|=\frac{1}{T}|u(T)-u(0)| \leq \frac{1}{T} \max \{|\alpha(T)-\beta(0)|,|\beta(T)-\alpha(0)|\} \Rightarrow\left|u^{\prime}\left(t_{2}\right)\right| \leq \zeta<M_{1}$.

Since $u \in C^{1}(\Omega)$, by the intermediate value theorem, there exists $t_{3}$ and $t_{4} \in\left[t_{2}, t_{1}\right)$ with $t_{3}<t_{4}$ such that $\left|u^{\prime}\left(t_{3}\right)\right|=\zeta$ and $\left|u^{\prime}\left(t_{4}\right)\right|=M_{1}$. We have:

$$
\begin{aligned}
-\left(\Phi\left(u^{\prime}(t)\right)\right)^{\prime} & =f\left(t, u(t), u^{\prime}(t)\right)+\Xi(u(t)) \text { a.e on } \Omega=[0, T] \\
\Rightarrow\left|\Phi\left(u^{\prime}(t)\right)\right|^{\prime} & \leq\left|\left(\Phi\left(u^{\prime}(t)\right)\right)^{\prime}\right| \leq\left|f\left(t, u(t), u^{\prime}(t)\right)\right|+|\Xi(u(t))| \\
& \leq \eta\left(\left|\Phi\left(u^{\prime}(t)\right)\right|\right)\left(\psi(t)+c\left|u^{\prime}(t)\right|\right)+|\Xi(u(t))| \text { a.e on } \Omega .
\end{aligned}
$$

Thus:

$$
\frac{\left|\Phi\left(u^{\prime}(t)\right)\right|^{\prime}}{\eta\left(\left|\Phi\left(u^{\prime}(t)\right)\right|\right)} \leq \psi(t)+c\left|u^{\prime}(t)\right|+\frac{|\Xi(u(t))|}{\eta\left(\left|\Phi\left(u^{\prime}(t)\right)\right|\right)} \text { a.e on }\left[t_{3}, t_{4}\right]
$$

and then

$$
\int_{t_{3}}^{t_{4}} \frac{\left|\Phi\left(u^{\prime}(t)\right)\right|^{\prime}}{\eta\left(\left|\Phi\left(u^{\prime}(t)\right)\right|\right)} \mathrm{d} t \leq\|\psi\|_{1}+c\left(\max _{\Omega} \beta-\min _{\Omega} \alpha\right)+\mu T
$$

Setting $s=\left|\Phi\left(u^{\prime}(t)\right)\right|$, we have:

$$
\int_{\Phi(\zeta)}^{\Phi\left(M_{1}\right)} \frac{\mathrm{d} s}{\eta(s)} \leq\|\psi\|_{1}+c\left(\max _{\Omega} \beta-\min _{\Omega} \alpha\right)+\mu T
$$


which contradicts the choice of $M_{1}>0$.

Now, we introduce the truncation map: $\varrho: \Omega \times \mathbb{R} \times \mathbb{R} \rightarrow \mathbb{R}^{2}$ defined by

$$
\rho(t, x, y)= \begin{cases}\left(\alpha(t), \alpha^{\prime}(t)\right) & \text { if } x<\alpha(t) \\ \left(\beta(t), \beta^{\prime}(t)\right) & \text { if } x>\beta(t) \\ \left(x, M_{0}\right) & \text { if } \alpha(t) \leq x \leq \beta(t), y>M_{0} \\ \left(x,-M_{0}\right) & \text { if } \alpha(t) \leq x \leq \beta(t), y<-M_{0} \\ (x, y) & \text { if } \alpha(t) \leq x \leq \beta(t),|y| \leq M_{0}\end{cases}
$$

where $M_{0}>\max \left\{M_{1},\left\|\alpha^{\prime}\right\|_{\infty},\left\|\beta^{\prime}\right\|_{\infty}\right\}$ and the penalty function $\Lambda: \Omega \times \mathbb{R} \rightarrow \mathbb{R}$ defined by:

$$
\Lambda(t, x)= \begin{cases}\Phi_{p}(\alpha(t))-\Phi_{p}(x) & \text { if } x<\alpha(t) \\ 0 & \text { if } \alpha(t) \leq x \leq \beta(t) \\ \Phi_{p}(\beta(t))-\Phi_{p}(x) & \text { if } x>\beta(t)\end{cases}
$$

We set $f_{1}(t, x, y)=f(t, \varrho(t, x, y))$. Note that for ae $x \in[\alpha(t), \beta(t)]$ and all $|y|<M_{0}$, we have $f_{1}(t, x, y)=f(t, x, y)$. Moreover, for almost all $t \in \Omega$ and all $x, y \in \mathbb{R}$, we have: $\left|f_{1}(t, x, y)\right| \leq \gamma_{r}(t)$ with $r=\max \left\{M_{0},\|\alpha\|_{\infty},\|\beta\|_{\infty}\right\}$. For every $u \in W^{1, p}((0, T))$, set

$$
N_{1}(u)(.)=f_{1}\left(., u(.), u^{\prime}(.)\right)
$$

and

$$
\hat{\Lambda}(u)(.)=\Lambda(., u(.))
$$

the Nemitsky operators corresponding to $f_{1}$ and $\Lambda$ respectively. We set $G(u)=N_{1}(u)+\hat{\Lambda}(u)$ for every $u \in W^{1, p}((0, T))$.

Proposition 12. If hypothesis $H(f)$ (ii) holds, then: $G: W^{1, p}((0, T)) \rightarrow L^{q}(\Omega)$ is continuous.

Proof. Since $N_{1}$ and $\hat{\Lambda}$ are Nemitsky operators, it is standard to show that they are continuous. It follows that $G$ is continuous.

We introduce the set

$$
D=\left\{u \in C^{1}(\Omega): \Phi\left(u^{\prime}\right) \in W^{1, q}(0, T), u^{\prime}(0) \in B_{1}(u(0)) \text { and }-u^{\prime}(T) \in B_{2}(u(T))\right\}
$$

and then we define the non-linear operator: $\vartheta: D \subseteq L^{p}(\Omega) \rightarrow L^{q}(\Omega)$ by

$$
\vartheta(u)(.)=-\left(\Phi\left(u^{\prime}(.)\right)\right)^{\prime} \quad \text { for all } u \in D .
$$

Proposition 13. If the hypotheses $\left(H_{B}\right)$ and $\left(H_{\Phi}\right)$ hold, then $\vartheta$ is maximal monotone.

Proof. Given $h \in L^{q}(\Omega)$, we consider the following nonlinear boundary value problem:

$$
\left\{\begin{array}{l}
-\left(\Phi\left(u^{\prime}(t)\right)\right)^{\prime}+\Phi_{p}(u(t))=h(t) \text { a.e on } \Omega=[0, T] \\
u^{\prime}(0) \in B_{1}(u(0)),-u^{\prime}(T) \in B_{2}(u(T)) .
\end{array}\right.
$$

We show that problem (10) has a unique solution $u \in C^{1}(\Omega)$. To this end, 
given $v, w \in \mathbb{R}$, we consider the following two-point boundary value problem:

$$
\left\{\begin{array}{l}
-\left(\Phi\left(u^{\prime}(t)\right)\right)^{\prime}+\Phi_{p}(u(t))=h(t) \text { a.e on } \Omega=[0, T] \\
u(0)=v, u(T)=w
\end{array}\right.
$$

We set $\gamma(t)=\left(1-\frac{t}{T}\right) v+\frac{t}{T} w$. Then $\gamma(0)=v$ and $\gamma(T)=w$. We consider the function $y$ defined by $y(t)=u(t)-\gamma(t)$ and rewrite (11) in the terms of this function.

$$
\left\{\begin{array}{l}
-\left(\Phi\left(y^{\prime}(t)+\gamma^{\prime}(t)\right)\right)^{\prime}+\Phi_{p}(y(t)+\gamma(t))=h(t) \text { a.e on } \Omega=[0, T] \\
y(0)=y(T)=0 .
\end{array}\right.
$$

This is a homogeneous Dirichlet problem for (11). To solve (12), let $V_{1}$ be the non-linear operator defined by: $V_{1}: W_{0}^{1, p}(\Omega) \rightarrow W^{-1, q}(\Omega)$

$$
\begin{aligned}
\left\langle V_{1}(y), z\right\rangle_{0}= & \int_{0}^{T} \Phi\left(y^{\prime}(t)+\gamma^{\prime}(t)\right) z^{\prime}(t) \mathrm{d} t \\
& +\int_{0}^{T} \Phi_{p}(y(t)+\gamma(t)) z(t) \mathrm{d} t, \forall y, z \in W_{0}^{1, p}(\Omega)
\end{aligned}
$$

where \langle\rangle$_{0}$ denote the duality brackets for the pair $\left(W^{-1, q}(\Omega), W_{0}^{1, p}(\Omega)\right)$.

Let us show that $V_{1}$ is strictly monotone.

Let $y, z \in W_{0}^{1, p}(\Omega)$. We have

$$
\begin{aligned}
&\left\langle V_{1}(y)-V_{1}(z), y-z\right\rangle_{0} \\
&=\left\langle V_{1}(y), y-z\right\rangle_{0}-\left\langle V_{1}(z), y-z\right\rangle_{0} \\
&=\int_{0}^{T} \Phi\left(y^{\prime}(t)+\gamma^{\prime}(t)\right)\left(y^{\prime}(t)-z^{\prime}(t)\right) \mathrm{d} t+\int_{0}^{T} \Phi_{p}(y(t)+\gamma(t))(y(t)-z(t)) \mathrm{d} t \\
&-\int_{0}^{T} \Phi\left(z^{\prime}(t)+\gamma^{\prime}(t)\right)\left(y^{\prime}(t)-z^{\prime}(t)\right) \mathrm{d} t-\int_{0}^{T} \Phi_{p}(z(t)+\gamma(t))(y(t)-z(t)) \mathrm{d} t .
\end{aligned}
$$

Then

$$
\begin{aligned}
& \left\langle V_{1}(y)-V_{1}(z), y-z\right\rangle_{0} \\
& =\int_{0}^{T}\left(\Phi\left(y^{\prime}(t)+\gamma^{\prime}(t)\right)-\Phi\left(z^{\prime}(t)+\gamma^{\prime}(t)\right)\right)\left(\left(y^{\prime}(t)+\gamma^{\prime}(t)\right)-\left(z^{\prime}(t)+\gamma^{\prime}(t)\right)\right) \mathrm{d} t \\
& \quad+\int_{0}^{T}\left(\Phi_{p}(y(t)+\gamma(t))-\Phi_{p}(z(t)+\gamma(t))\right)((y(t)+\gamma(t))-(z(t)+\gamma(t))) \mathrm{d} t .
\end{aligned}
$$

We know that $\Phi$ is monotone. Moreover, it is easy to show that $\Phi_{p}$ is strictly monotone. Whence

$$
\left\langle V_{1}(y)-V_{1}(z), y-z\right\rangle_{0}>0 .
$$

Therefore, $V_{1}$ is strictly monotone.

- Let us show that $V_{1}$ is demicontinuous.

Using the extended dominated convergence theorem (see for example Hu-Papageorgiou 10, Theorem A.2.54, p. 907), it follows easily that $V_{1}$ is demicontinuous.

Recall that an operator monotone and demicontinuous is maximal monotone.

So $V_{1}$ is maximal monotone.

Let us show that $V_{1}$ is coercive. 
For $y \in W_{0}^{1, p}(\Omega)$ we have:

$$
\begin{aligned}
& \left\langle V_{1}(y), y\right\rangle_{0}=\int_{0}^{T} \Phi\left(y^{\prime}(t)+\gamma^{\prime}(t)\right) y^{\prime}(t) \mathrm{d} t+\int_{0}^{T} \Phi_{p}(y(t)+\gamma(t)) y(t) \mathrm{d} t \\
& =\int_{0}^{T} \Phi\left(y^{\prime}(t)+\gamma^{\prime}(t)\right)\left(y^{\prime}(t)+\gamma^{\prime}(t)\right) \mathrm{d} t-\int_{0}^{T} \Phi\left(y^{\prime}(t)+\gamma^{\prime}(t)\right) \gamma^{\prime}(t) \mathrm{d} t \\
& +\int_{0}^{T} \Phi_{p}(y(t)+\gamma(t))(y(t)+\gamma(t)) \mathrm{d} t-\int_{0}^{T} \Phi_{p}(y(t)+\gamma(t)) \gamma(t) \mathrm{d} t \\
& \geq \int_{0}^{T} \Phi\left(y^{\prime}(t)+\gamma^{\prime}(t)\right)\left(y^{\prime}(t)+\gamma^{\prime}(t)\right) \mathrm{d} t-\int_{0}^{T}\left|\Phi\left(y^{\prime}(t)+\gamma^{\prime}(t)\right)\right| \gamma^{\prime}(t) \mid \mathrm{d} t \\
& +\int_{0}^{T} \Phi_{p}(y(t)+\gamma(t))(y(t)+\gamma(t)) \mathrm{d} t-\int_{0}^{T}\left|\Phi_{p}(y(t)+\gamma(t))\right| \gamma(t) \mid \mathrm{d} t .
\end{aligned}
$$

Hence, using the hypotheses (b) and (c) on $\Phi$, we obtain:

$$
\begin{aligned}
&\left\langle V_{1}(y), y\right\rangle_{0} \geq \int_{0}^{T}\left(|(y(t)+\gamma(t))|^{p}+d_{1}\left|\left(y^{\prime}(t)+\gamma^{\prime}(t)\right)\right|^{p}\right) \mathrm{d} t \\
&-c_{1} \int_{0}^{T}\left(d_{2}+|(y(t)+\gamma(t))|^{p-1}+d_{3}\left|\left(y^{\prime}(t)+\gamma^{\prime}(t)\right)\right|^{p-1}\right) \mathrm{d} t \\
& \text { with } c_{1}=\max \left\{|v|+|w|, \frac{|v-w|}{T}\right\} .
\end{aligned}
$$

whence:

$$
\left\langle V_{1}(y), y\right\rangle_{0} \geq \eta_{1}\|y+\gamma\|^{p}-\eta_{2}\|y+\gamma\|^{p-1}-\eta_{3} \text {, for some } \eta_{1}, \eta_{2}, \eta_{3}>0 .
$$

Therefore, $V_{1}$ is coercive.

Recall that an operator maximal monotone which is coercive is surjective.

Moreover, since $V_{1}$ is strictly monotone, we infer that there exists a unique $y \in W_{0}^{1, p}((0, T))$ such that $V_{1}(y)=h$. It follows easily that $y \in C^{1}(\Omega)$ and it solves the problem (12). Then $u=y+\gamma \in C^{1}(\Omega)$ and it is the solution of the problem (11). We can define the solution map $\sigma: \mathbb{R} \times \mathbb{R} \rightarrow C^{1}(\Omega)$ which to each pair $(v, w)$ assigns the unique solution of the problem (11). Let $\Delta: \mathbb{R} \times \mathbb{R} \rightarrow \mathbb{R} \times \mathbb{R}$ be defined by:

$$
\Delta(v, w)=\left(-\Phi\left(\sigma(v, w)^{\prime}(0)\right), \Phi\left(\sigma(v, w)^{\prime}(T)\right)\right) .
$$

We claim that $\Delta$ is monotone.

Indeed, for $\left(v_{1}, w_{1}\right),\left(v_{2}, w_{2}\right) \in \mathbb{R}^{2}$, we have:

$$
\begin{aligned}
&\left\langle\Delta\left(v_{1}, w_{1}\right)-\Delta\left(v_{2}, w_{2}\right),\left(v_{1}, w_{1}\right)-\left(v_{2}, w_{2}\right)\right\rangle_{2} \\
&=\left\langle-\left(\Phi\left(u_{1}^{\prime}(0)\right)-\Phi\left(u_{2}^{\prime}(0)\right), \Phi\left(u_{1}^{\prime}(T)\right)-\Phi\left(u_{2}^{\prime}(T)\right)\right),\right. \\
&\left.\left(u_{1}(0)-u_{2}(0), u_{1}(T)-u_{2}(T)\right)\right\rangle_{2} \\
&=\left(\Phi\left(u_{2}^{\prime}(0)\right)-\Phi\left(u_{1}^{\prime}(0)\right)\right)\left(u_{1}(0)-u_{2}(0)\right) \\
&+\left(\Phi\left(u_{1}^{\prime}(T)\right)-\Phi\left(u_{2}^{\prime}(T)\right)\right)\left(u_{1}(T)-u_{2}(T)\right) \\
&= \int_{0}^{T}\left(\Phi\left(u_{1}^{\prime}(t)\right)\right)^{\prime}-\left(\Phi\left(u_{2}^{\prime}(t)\right)\right)^{\prime}\left(u_{1}(t)-u_{2}(t)\right) \mathrm{d} t \\
&+\int_{0}^{T}\left(\Phi\left(u_{1}^{\prime}(t)\right)-\Phi\left(u_{2}^{\prime}(t)\right)\right)\left(u_{1}^{\prime}(t)-u_{2}^{\prime}(t)\right) \mathrm{d} t
\end{aligned}
$$

where \langle\rangle$_{2}$ is the scalar product in $\mathbb{R}^{2}$.

From (11), we have $\left(\Phi\left(u_{1}^{\prime}(t)\right)\right)^{\prime}-\left(\Phi\left(u_{2}^{\prime}(t)\right)\right)^{\prime}=\Phi_{p}\left(u_{1}(t)\right)-\Phi_{p}\left(u_{2}(t)\right)$. Because of monotonicity of the operators $\Phi$ and $\Phi_{p}$, we have the monotonicity of 
$\Delta$.

We claim that $\Delta$ is continuous.

Indeed, let $\left(b_{n}\right)_{n \geq 1}$ and $\left(e_{n}\right)_{n \geq 1}$ be real sequences converging respectively to $b$ and $e$.

Let us set that

$$
\begin{aligned}
& u_{n}=\sigma\left(b_{n}, e_{n}\right), u=\sigma(b, e), \gamma_{n}(t)=\left(1-\frac{t}{T}\right) b_{n}+\frac{t}{T} e_{n}, \\
& \gamma(t)=\left(1-\frac{t}{T}\right) b+\frac{t}{T} e \text { and } y_{n}=u_{n}-\gamma_{n}, \text { for all } n \geq 1 .
\end{aligned}
$$

Now, we consider the following sequence of problems:

$$
\left\{\begin{array}{l}
-\left(\Phi\left(y_{n}^{\prime}(t)+\gamma_{n}^{\prime}(t)\right)\right)^{\prime}+\Phi_{p}\left(y_{n}(t)+\gamma_{n}(t)\right)=h(t) \text { a.e on } \Omega=[0, T] \\
y_{n}(0)=y_{n}(T)=0 .
\end{array}\right.
$$

We claim that $\left\{u_{n}=y_{n}+\gamma_{n}\right\}_{n \geq 1} \subseteq W^{1, p}(\Omega)$ is bounded.

Let us multiply (13) by $y_{n}$ and integrate on $\Omega$, we obtain:

$$
\begin{aligned}
& \int_{0}^{T}-\left(\Phi\left(y_{n}^{\prime}(t)+\gamma_{n}^{\prime}(t)\right)\right)^{\prime} y_{n}(t) \mathrm{d} t+\int_{0}^{T}\left(\Phi_{p}\left(y_{n}(t)+\gamma_{n}(t)\right)\right) y_{n}(t) \mathrm{d} t \\
& =\int_{0}^{T} h(t) y_{n}(t) \mathrm{d} t .
\end{aligned}
$$

By using green's formula, we obtain:

$$
\begin{aligned}
& \int_{0}^{T}\left(\Phi\left(y_{n}^{\prime}(t)+\gamma_{n}^{\prime}(t)\right)\right)^{\prime} y_{n}^{\prime}(t) \mathrm{d} t+\int_{0}^{T}\left(\Phi_{p}\left(y_{n}(t)+\gamma_{n}(t)\right)\right) y_{n}(t) \mathrm{d} t \\
& =\int_{0}^{T} h(t) y_{n}(t) \mathrm{d} t \\
& \int_{0}^{T}\left(\Phi\left(y_{n}^{\prime}(t)+\gamma_{n}^{\prime}(t)\right)\right)\left(y_{n}^{\prime}(t)+\gamma_{n}^{\prime}(t)\right) \mathrm{d} t \\
& \quad+\int_{0}^{T}\left(\Phi_{p}\left(y_{n}(t)+\gamma_{n}(t)\right)\right)\left(y_{n}(t)+\gamma_{n}(t)\right) \mathrm{d} t \\
& \quad-\int_{0}^{T}\left(\Phi\left(y_{n}^{\prime}(t)+\gamma_{n}^{\prime}(t)\right)\right) \gamma_{n}^{\prime}(t) \mathrm{d} t \\
& -\int_{0}^{T}\left(\Phi_{p}\left(y_{n}(t)+\gamma_{n}(t)\right)\right) \gamma_{n}(t) \mathrm{d} t \\
& =\int_{0}^{T} h(t) y_{n}(t) \mathrm{d} t \\
& \geq \int_{0}^{T}\left(\Phi\left(y_{n}^{\prime}(t)+\gamma_{n}^{\prime}(t)\right)\right){ }^{\prime}\left(y_{n}^{\prime}(t)+\gamma_{n}^{\prime}(t)\right) \mathrm{d} t \\
& \quad+\int_{0}^{T}\left(\Phi_{p}\left(y_{n}(t)+\gamma_{n}(t)\right)\right)\left(y_{n}(t)+\gamma_{n}(t)\right) \mathrm{d} t \\
& \quad-\int_{0}^{T}\left|\left(\Phi\left(y_{n}^{\prime}(t)+\gamma_{n}^{\prime}(t)\right)\right)^{\prime}\right|\left|\gamma_{n}^{\prime}(t)\right| \mathrm{d} t \\
& \quad-\int_{0}^{T}\left|\left(\Phi_{p}\left(y_{n}(t)+\gamma_{n}(t)\right)\right)\right|\left|\gamma_{n}(t)\right| \mathrm{d} t \\
& \geq \int_{0}^{T}\left|\left(y_{n}(t)+\gamma_{n}(t)\right)\right|^{p}+d_{1} \mid\left(y_{n}^{\prime}(t)+\left.\gamma_{n}^{\prime}(t)\right|^{p} \mathrm{~d} t\right. \\
& \quad-c_{2} \int_{0}^{T}\left(d_{2}+\left|\left(y_{n}(t)+\gamma_{n}(t)\right)\right|^{p-1}+d_{3}\left|\left(y_{n}^{\prime}(t)+\gamma_{n}^{\prime}(t)\right)\right|^{p-1}\right) \mathrm{d} t \\
& \quad w i t h \quad c_{2}=\max _{n \geq 1}\left\{\left|v_{n}\right|+\left|w_{n}\right|, \frac{\left|v_{n}-w_{n}\right|}{T}\right\} .
\end{aligned}
$$


whence:

$$
\int_{0}^{T} h(t) y_{n}(t) \mathrm{d} t \geq \eta_{1}^{\prime}\left\|y_{n}+\gamma_{n}\right\|^{P}-\eta_{2}^{\prime}\left\|y_{n}+\gamma_{n}\right\|^{p-1}-\eta_{3}^{\prime} \text { for some } \eta_{1}^{\prime}, \eta_{2}^{\prime}, \eta_{3}^{\prime}>0 .
$$

Furthermore, using the Cauchy-Schwartz inequality and then the triangular inequality, we obtain the following inequalities:

$$
\begin{aligned}
\int_{0}^{T} h(t) y_{n}(t) \mathrm{d} t & \leq\|h\|_{q}\left\|y_{n}\right\|_{p} \leq\|h\|_{q}\left(\left\|y_{n}+\gamma_{n}\right\|_{p}+\left\|\gamma_{n}\right\|_{p}\right) \\
& \leq\|h\|_{q}\left(\left\|y_{n}+\gamma_{n}\right\|+\left\|\gamma_{n}\right\|_{p}\right) .
\end{aligned}
$$

Then:

$$
\eta_{1}^{\prime}\left\|y_{n}+\gamma_{n}\right\|^{p} \leq \eta^{\prime}\left\|y_{n}+\gamma_{n}\right\|^{p-1}+\|h\|_{q}\left\|y_{n}+\gamma_{n}\right\|+\|h\|_{q}\left\|\gamma_{n}\right\|_{p}+\eta_{3}^{\prime} .
$$

So

$$
\eta_{4}\left\|y_{n}+\gamma_{n}\right\|^{p} \leq \eta_{5}\left\|y_{n}+\gamma_{n}\right\|^{p-1}+\eta_{6}\left\|y_{n}+\gamma_{n}\right\|_{p}+\eta_{7} \text { for some } \eta_{4}, \eta_{5}, \eta_{6}, \eta_{7}>0 \text {. }
$$

Therefore, the sequence $\left\{u_{n}=y_{n}+\gamma_{n}\right\}_{n \geq 1} \subseteq W^{1, p}(\Omega)$ is bounded. It follows immediately that the sequences $\left\{\Phi_{p}\left(u_{n}\right)\right\}_{n \geq 1} \subseteq L^{q}(\Omega)$ is bounded. So directly from the problem (11), we get that the sequence $\left\{\left(\Phi\left(u_{n}^{\prime}\right)\right)^{\prime}\right\}_{n \geq 1} \subseteq L^{q}(\Omega)$ is bounded. By integration, we obtain $\left\{\Phi\left(u_{n}^{\prime}\right)\right\}_{n \geq 1} \subseteq L^{q}(\Omega)$. So the sequence $\left\{\Phi\left(u_{n}^{\prime}\right)\right\}_{n \geq 1} \subseteq W^{1, q}((0, T))$ is bounded. Then we have respectively

$$
u_{n} \rightarrow u \text { in } W^{1, p}(\Omega), \Phi_{p}\left(u_{n}\right) \rightarrow v \text { in } L^{q}(0, T) \text { and } \Phi\left(u_{n}^{\prime}\right) \rightarrow w \text { in } W^{1, q}((0, T))
$$

Due to the compact embedding of $W^{1, p}((0, T))$ in $C(\Omega)$, we have:

$$
u_{n} \rightarrow u \text { in } C(\Omega) \text { and } \Phi\left(u_{n}^{\prime}\right) \rightarrow w \text { in } C(\Omega) .
$$

Since $\Phi$ is an increasing homeomorphism, $\Phi^{-1}$ exists and is continuous. So, we have: $u_{n}^{\prime} \rightarrow \Phi^{-1}(w)$ in $C(\Omega)$. Whence $u^{\prime}=\Phi^{-1}(w) \quad\left(\right.$ i.e. $\left.\Phi\left(u^{\prime}\right)=w\right)$. Therefore passing to the limit as $n \rightarrow+\infty$, we have:

$$
\left\{\begin{array}{l}
-\left(\Phi\left(u^{\prime}(t)\right)\right)^{\prime}+\Phi_{p}(u(t))=h(t) \text { a.e on } \Omega=[0, T] \\
u(0)=b, u(T)=e
\end{array}\right.
$$

$\Rightarrow u=\sigma(a, e)$ (i.e. $\sigma: \mathbb{R} \times \mathbb{R} \rightarrow C^{1}(\Omega)$ is continuous). So, $\Delta$ is continuous.

We claim that $\Delta$ is coercive.

For $(v, w) \in \mathbb{R}^{2}$, we have:

$$
\begin{aligned}
& \frac{\langle\Delta(v, w),(v, w)\rangle_{2}}{\|(v, w)\|_{\mathbb{R}^{2}}}=\frac{\Phi\left(u^{\prime}(T)\right) u(T)-\Phi\left(u^{\prime}(0)\right) u(0)}{\|(v, w)\|_{\mathbb{R}^{2}}} \\
& =\frac{\int_{0}^{T}\left(\Phi\left(u^{\prime}(t)\right)\right)^{\prime} u(t) \mathrm{d} t+\int_{0}^{T} \Phi\left(u^{\prime}(t)\right) u^{\prime}(t) \mathrm{d} t}{\|(v, w)\|_{\mathbb{R}^{2}}} \\
& \frac{\int_{0}^{T}\left(\Phi\left(u^{\prime}(t)\right)\right) u(t) \mathrm{d} t+\int_{0}^{T} \Phi\left(u^{\prime}(t)\right) u^{\prime}(t) \mathrm{d} t}{\|(v, w)\|_{\mathbb{R}^{2}}} \\
& =\frac{\int_{0}^{T} \Phi_{p}(u(t)) u(t) \mathrm{d} t-\int_{0}^{T} h(t) u(t) \mathrm{d} t+\int_{0}^{T} \Phi\left(u^{\prime}(t)\right) u^{\prime}(t) \mathrm{d} t}{\|(v, w)\|_{\mathbb{R}^{2}}}
\end{aligned}
$$




$$
\begin{aligned}
& \geq \frac{\int_{0}^{T}\left(|u(t)|^{p}+d_{1}\left|u^{\prime}(t)\right|^{p}\right) \mathrm{d} t-\left(\int_{0}^{T}|h(t)|^{q}\right)^{\frac{1}{q}}\left(\int_{0}^{T}|u(t)|^{p}\right)^{\frac{1}{p}}}{\|(v, w)\|_{\mathbb{R}^{2}}} \\
& \geq \frac{d_{4}\left(\|u\|_{p}^{p}+\left\|u^{\prime}\right\|_{p}^{p}\right)-\|h\|_{q}\|u\|_{p}}{\|(v, w)\|_{\mathbb{R}^{2}}} .
\end{aligned}
$$

Then

$$
\frac{\langle\Delta(v, w),(v, w)\rangle_{2}}{\|(v, w)\|_{\mathbb{R}^{2}}} \geq \frac{d_{4}\left(\|u\|_{p}^{p}+\left\|u^{\prime}\right\|_{p}^{p}\right)-\|h\|_{q}\|u\|_{p}}{\|(v, w)\|_{\mathbb{R}^{2}}} \text { with } d_{4}=\min \left\{1, d_{1}\right\}
$$

where \|\|$_{\mathbb{R}^{2}}$ denote the euclidean norm in $\mathbb{R}^{2}$.

Since $u \in W^{1, p}(\Omega)$, by mean value theorem, there exists $t_{0} \in(0, T)$ such that $\left|u\left(t_{0}\right)\right| T=\int_{0}^{T}|u(t)| \mathrm{d} t$.

As $u(t)-u\left(t_{0}\right)=\int_{t_{0}}^{t} u^{\prime}(s) \mathrm{d} s$, we have:

$$
|u(t)| \leq\left|u\left(t_{0}\right)\right|+\int_{0}^{T}\left|u^{\prime}(s)\right| \mathrm{d} s \leq \frac{1}{T}\|u\|_{1}+T^{\frac{1}{q}}\left\|u^{\prime}\right\|_{P} \leq \frac{T^{\frac{1}{q}}}{T}\|u\|_{P}+T^{\frac{1}{q}}\left\|u^{\prime}\right\|_{P}
$$

for all $t \in \Omega$. In particular, we have:

$$
\begin{aligned}
\|(v, w)\|_{\mathbb{R}^{2}} & =\sqrt{u^{2}(0)+u^{2}(T)} \leq \sqrt{2}\left(\frac{T^{\frac{1}{q}}}{T}\|u\|_{P}+T^{\frac{1}{q}}\left\|u^{\prime}\right\|_{P}\right) \\
& \leq \sqrt{2} \max \left\{\frac{T^{\frac{1}{q}}}{T}, T^{\frac{1}{q}}\right\}\left(\|u\|_{P}+\left\|u^{\prime}\right\|_{P}\right) .
\end{aligned}
$$

Hence

$$
\frac{\langle\Delta(v, w),(v, w)\rangle_{2}}{\|(v, w)\|_{\mathbb{R}^{2}}} \geq \frac{d_{4}\left(\|u\|_{p}^{p}+\left\|u^{\prime}\right\|_{p}^{p}\right)-\|h\|_{q}\|u\|_{p}}{\varsigma\left(\|u\|_{p}+\left\|u^{\prime}\right\|_{p}\right)} \text { with } \varsigma=\sqrt{2} \max \left\{\frac{T^{\frac{1}{q}}}{T}, T^{\frac{1}{q}}\right\} .
$$

Therefore $\Delta$ is coercive.

We infer that $\Delta$ is maximal monotone (being continuous, monotone) and coercive. Thus $\Delta$ is surjective. Now, we consider $\theta=\Delta+B: \mathbb{R} \times \mathbb{R} \rightarrow P(\mathbb{R} \times \mathbb{R})$ with operator $B$ defined by $B(b, e)=\left(\Phi \circ B_{1}(b), \Phi \circ B_{2}(e)\right)$ for all $(b, e) \in \mathbb{R} \times \mathbb{R}$. Since $\Delta$ is coercive and $B$ is maximal monotone, we deduce that $\theta$ is coercive. Also, $\theta$ is maximal monotone (see Brezis [15] Corollary 2.7, p. 36 or Zeidler [14] Theorem 32.I, p. 897). So $\theta$ is surjective. We infer that we can find $(b, e) \in \mathbb{R} \times \mathbb{R}$ such that $(0,0) \in \theta(b, e)$. Since $(0,0) \in \theta(b, e)$, we can find $t, s \in \mathbb{R}$ such that $t \in \Phi \circ B_{1}(b)$ and $s \in \Phi \circ B_{2}(e)$ and $0=-\Phi\left(u^{\prime}(0)\right)+t \quad$ and $\quad 0=\Phi\left(u^{\prime}(T)\right)+s \quad$. So $\Phi(u(0)) \in \Phi \circ B_{1}(b) \quad$ and $-\Phi(u(T)) \in \Phi \circ B_{2}(e)$. It follows that $\left(u^{\prime}(0),-u^{\prime}(T)\right) \in\left(B_{1}(b), B_{2}(e)\right)$. Therefore $x_{0}=\sigma(b, e)$ is the solution of the problem (10).

Let $H: L^{p}(\Omega) \rightarrow L^{q}(\Omega)$ be the operator defined by: 


$$
H(u)(.)=\Phi_{p}(u(.)) .
$$

Since $\Phi_{p}$ is continuous and monotone, then $H$ is continuous and monotone. Therefore $H$ is maximal monotone. Moreover it is evident to see that $H$ is strictly monotone.

Since in (10) the choice of $h$ is arbitrary, then by the previous arguments, we have:

$$
R(\vartheta+H)=L^{q}(\Omega) \quad(\text { ie } \vartheta+H \text { is surjective }) .
$$

We denote by $\langle. . .\rangle_{p}$ the duality brackets between the pair $\left(L^{p}(\Omega), L^{q}(\Omega)\right)$.

Let us show that $\vartheta+H$ surjective implies $\vartheta$ is maximal monotone

For this purpose, we suppose that, for some $y \in L^{p}(\Omega)$ and some $v \in L^{q}(\Omega)$ :

$$
\langle\vartheta(u)-v, u-y\rangle_{p} \geq 0 \text { for all } u \in D .
$$

Because of (14), we can find $u_{1} \in D$ such that:

$$
\vartheta\left(u_{1}\right)+H\left(u_{1}\right)=v+H(y) .
$$

We use this in (16) with $u=u_{1}$, we obtain:

$$
\begin{gathered}
\left\langle\vartheta\left(u_{1}\right)-\vartheta\left(u_{1}\right)-H\left(u_{1}\right)+H(y), u_{1}-y\right\rangle_{p} \geq 0 \\
\Rightarrow\left\langle H(y)-H\left(u_{1}\right), u_{1}-y\right\rangle_{p} \geq 0
\end{gathered}
$$

Because $H$ is strictly monotone, from (16), we conclude that $y=u_{1} \in D$ and $v=\vartheta\left(u_{1}\right)$. So $\vartheta$ is maximal monotone. In addition, since $\vartheta$ is monotone, we have $\langle\vartheta(u)+H(u), u\rangle_{p} \geq\langle H(u), u\rangle_{2}=|u|^{p}$. Whence the operator

$\vartheta+H: D \subseteq L^{p}(\Omega) \rightarrow L^{q}(\Omega)$ is maximal monotone, strictly monotone and coercive. Therefore $\Psi=(\vartheta+H)^{-1}: L^{q}(\Omega) \rightarrow D \subseteq W^{1, p}((0, T))$ is well defined, single valued, and maximal monotone (From $L^{q}(\Omega)$ into $L^{p}(\Omega)$ ).

Proposition 14. If hypothesis $\left(H_{B}\right)$ holds, then

$L: L^{q}(\Omega) \rightarrow D \subseteq W^{1, p}((0, T))$ is completly continuous.

Proof. Suppose that $v_{n} \rightarrow v$ in $L^{q}(\Omega)$. We have to show that $L\left(v_{n}\right) \rightarrow L(v)$ in $W^{1, p}(\Omega)$. let us set $u_{n}=L\left(v_{n}\right)$ for all $n \geq 1$. We have

$$
\begin{gathered}
u_{n} \in D \text { and } \vartheta\left(u_{n}\right)+H\left(u_{n}\right)=v_{n} . \\
\Rightarrow\left\langle\vartheta\left(u_{n}\right), u_{n}\right\rangle_{p}+\left\langle H\left(u_{n}\right), u_{n}\right\rangle_{p}=\left\langle v_{n}, u_{n}\right\rangle_{p} .
\end{gathered}
$$

By integration by part, we obtain:

$$
\begin{aligned}
& -\Phi\left(u_{n}^{\prime}(T)\right) u_{n}(T)+\Phi\left(u_{n}^{\prime}(0)\right) u_{n}(0)+\int_{0}^{T} \Phi\left(u_{n}^{\prime}(t)\right) u_{n}^{\prime}(t) \mathrm{d} t \\
& +\int_{0}^{T} \Phi_{p}\left(u_{n}(t)\right) u_{n}(t) \mathrm{d} t=\left\langle v_{n}, u_{n}\right\rangle_{p} .
\end{aligned}
$$

Since $u_{n} \in D$, we have $u_{n}^{\prime}(0) \in B_{1}\left(u_{n}(0)\right)$ and $-u_{n}^{\prime}(T) \in B_{1}\left(u_{n}(T)\right)$ for all $n \geq 1$. We recall that $(0,0) \in \operatorname{Gr}\left(B_{i}\right), i=1,2$, then:

$$
u_{n}^{\prime}(0) u_{n}(0) \geq 0 \quad \text { and } \quad u_{n}^{\prime}(T) u_{n}(T) \leq 0 .
$$

Moreover, the map $\Phi$ being monotone, we have:

$$
\Phi\left(u_{n}^{\prime}(0)\right) u_{n}(0) \geq 0 \quad \text { and } \quad \Phi\left(u_{n}^{\prime}(T)\right) u_{n}^{\prime}(T) \geq 0 .
$$


From (19) and (20), we obtain:

$$
\Phi\left(u_{n}^{\prime}(0)\right) u_{n}(0) \geq 0 \quad \text { and } \quad \Phi\left(u_{n}^{\prime}(T)\right) u_{n}(T) \leq 0 .
$$

From (21) and (18), we infer that:

$$
\left\langle v_{n}, u_{n}\right\rangle_{p} \geq \int_{0}^{T} \Phi\left(u_{n}^{\prime}(t)\right) u_{n}^{\prime}(t) \mathrm{d} t+\int_{0}^{T} \Phi_{p}\left(u_{n}(t)\right) u_{n}(t) .
$$

By hypothesis $b$ ) on $\Phi$, we have:

$$
\int_{0}^{T} \Phi\left(u_{n}^{\prime}(t)\right) u_{n}^{\prime}(t) \mathrm{d} t+\int_{0}^{T} \Phi_{p}\left(u_{n}(t)\right) u_{n}(t) \geq \int_{0}^{T}\left(\left|u_{n}(t)\right|^{p}+d_{1}\left|u_{n}^{\prime}(t)\right|^{p}\right) \mathrm{d} t .
$$

It follows from (22) and (23) that:

$$
\left\langle v_{n}, u_{n}\right\rangle_{p} \geq \int_{0}^{T}\left(\left|u_{n}(t)\right|^{p}+d_{1}\left|u_{n}^{\prime}(t)\right|^{p}\right) \mathrm{d} t .
$$

whence:

$$
\left\|u_{n}\right\|^{p-1} \leq \eta_{7} \text { for some } \eta_{7}>0 .
$$

Therefore the sequence $\left\{u_{n}\right\}_{n>1} \subseteq W^{1, p}((0, T))$ is bounded. Then we can find a convergente subsequence of $\left\{u_{n}\right\}_{n \geq 1}$. So $u_{n} \rightarrow u$ in $W^{1, p}((0, T))$. Due to the compact embedding of $W^{1, p}((0, T))$ in $C(\Omega)$, we have $u_{n} \rightarrow u$ in $C(\Omega)$. Since $\left\{u_{n}\right\}_{n \geq 1} \subseteq W^{1, p}((0, T))$ is bounded, we have $\left\{u_{n}^{\prime}\right\}_{n \geq 1} \subseteq L^{p}(\Omega)$ and $\left\{u_{n}\right\}_{n \geq 1} \subseteq L^{p}(\Omega)$ are bounded. It follows immediatly that: $\left\{\Phi_{p}\left(u_{n}\right)\right\}_{n \geq 1} \subseteq L^{q}(\Omega)$ is bounded. Then $\vartheta\left(u_{n}\right)+H\left(u_{n}\right)=v_{n}$ imply that $\left\{\left(\Phi\left(u_{n}^{\prime}\right)\right)^{\prime}\right\}_{n \geq 1} \subseteq L^{q}(\Omega)$ is bounded. Whence, by integration, $\left\{\Phi\left(u_{n}^{\prime}\right)\right\}_{n \geq 1} \subseteq W^{1, q}((0, T))$ is bounded. So we can suppose that $\Phi\left(u_{n}^{\prime}\right) \rightarrow h$ in $W^{1, q}((0, T))$. Due to the compact embedding of $W^{1, q}(0, T)$ in $C(\Omega)$, we obtain $\Phi\left(u_{n}^{\prime}\right) \rightarrow h$ in $C(\Omega)$. Since $\Phi$ is an increasing homeomorphism, $\Phi^{-1}$ exists and is continuous. So, we have $\Phi^{-1}\left(\Phi\left(u_{n}^{\prime}(t)\right)\right) \rightarrow \Phi^{-1}(h(t))$ for all $t \in \Omega$. Then $u_{n}^{\prime}(t) \rightarrow \Phi^{-1}(h(t))$ for all $t \in \Omega$. It follows that $u_{n}^{\prime}(.) \rightarrow \Phi^{-1}(h()$.$) in L^{P}(\Omega)$ (By Lebesgue dominated convergence theorem). We infer that: $u^{\prime}=\Phi^{-1}(h()$.$) . It follows h=\Phi\left(u^{\prime}\right)$

$$
\begin{aligned}
& \Phi\left(u_{n}^{\prime}\right) \rightarrow \Phi\left(u^{\prime}\right) \text { in } C(\Omega) \\
& \Rightarrow u_{n}^{\prime} \rightarrow u^{\prime} \text { in } L^{p}(\Omega) .
\end{aligned}
$$

But recall that $u_{n} \rightarrow u$ in $L^{p}(\Omega)$. Thus $u_{n} \rightarrow u$ in $W^{1, p}((0, T))$. This prove that the operator $L$ is completly continuous.

Proposition 15. If the conditions in lemma 1 hold, then a function $u \in C^{1}(\Omega)$ is solution of (1) if and only if $u \in D$ and $u$ is a fixed point of $K: C^{1}(\Omega) \rightarrow D \subseteq C^{1}(\Omega)$ defined by:

$$
\begin{gathered}
K(u)=L \circ Q(u) \text { with the operator } Q \text { defined by: } \\
Q(u)=N(u)+\hat{\Xi}(u)+H(u) .
\end{gathered}
$$

Moreover, for all $u \in C^{1}(\Omega),\left\|(K(u))^{\prime}\right\|_{\infty}<M_{0}$ and $K$ is continuous and completly continuous.

Proof. If $u$ is a solution of (1), then $\left(\Phi\left(u^{\prime}\right)\right)^{\prime} \in L^{q}(\Omega)$ because of hypothesis 
$\left(H_{f}\right)$ (iii). It follows that $\Phi\left(u^{\prime}\right) \in L^{q}(\Omega)$. So, $\Phi\left(u^{\prime}\right) \in W^{1, q}(\Omega)$. We have also $u^{\prime}(0) \in B_{1}(u(0))$ and $-u^{\prime}(T) \in B_{2}(u(T))$. Hence, $u \in D$.

Furthermore, we have:

$$
\begin{aligned}
& -\left(\Phi\left(u^{\prime}(t)\right)\right)^{\prime}=f\left(t, u(t), u^{\prime}(t)\right)+\Xi(u(t)) \text { a.e on }[0, T] \\
& \Leftrightarrow \vartheta(u)(t)=N(u)(t)+\hat{\Xi}(u)(t) \text { a.e on }[0, T] \\
& \Leftrightarrow \vartheta(u)(t)+H(u)(t)=N(u)(t)+\hat{\Xi}(u)(t)+H(u)(t) \text { a.e on }[0, T] \\
& \Leftrightarrow u(t)=L \circ Q(u)(t)=K(u)(t)
\end{aligned}
$$

Therefore, $u$ is a fixed point of $K$.

On the other hand, if $u \in D$ and $u$ is a fixed point of $K$, then we have $u \in C^{1}(\Omega), \quad u^{\prime}(0) \in B_{1}(u(0)), \quad-u^{\prime}(T) \in B_{2}(u(T))$ and $K(u)(t)=u(t)$ a.e on $[0, T] \Leftrightarrow-\left(\Phi\left(u^{\prime}(t)\right)\right)^{\prime}=f\left(t, u(t), u^{\prime}(t)\right)+\Xi(u(t))$ a.e on $[0, T]$. Hence, $u$ is solution of $(1)$.

Finally, by lemma 1, we have: $\forall u \in C^{1}(\Omega),\left\|(K(u))^{\prime}\right\|_{\infty} \leq M_{1}<M_{0}$.

- Let us show that $K$ is continuous.

Let $u_{n} \rightarrow u$ in $C^{1}(\Omega)$. Then, there exists $R>0$ such that $u_{n} \in \overline{B_{C^{1}}(R)}$ and $u \in \overline{B_{C^{1}}(R)}$.

We will show that $K\left(u_{n}\right) \rightarrow K(u)$ in $C^{1}(\Omega)$. That's mean $K\left(u_{n}\right) \rightarrow K(u)$ in $C(\Omega)$ and $\left(K\left(u_{n}\right)\right)^{\prime} \rightarrow(K(u))^{\prime}$ in $C(\Omega)$. For $n \geq 1$, we have:

$$
\begin{aligned}
& -\left(\Phi\left(u_{n}^{\prime}(t)\right)\right)^{\prime}=f\left(t, u_{n}(t), u_{n}^{\prime}(t)\right)+\Xi\left(u_{n}(t)\right) \text { a.e } t \in[0, T] \\
& \Leftrightarrow u_{n}^{\prime}(t)=\Phi^{-1} \circ\left(\Phi\left(u_{n}^{\prime}(0)\right)-\int_{0}^{t} f\left(s, u_{n}(s), u_{n}^{\prime}(s)\right)+\Xi\left(u_{n}(s)\right) \text { ds }\right) \text { a.e } t \in[0, T] \\
& \Leftrightarrow u_{n}^{\prime}(t)=\left(K\left(u_{n}\right)\right)^{\prime}(t) \text { a.e } t \in[0, T]
\end{aligned}
$$

Since $\Phi$ and $N$ are continuous respectively in $L^{q}(\Omega)$ and $C(\Omega)$, we have:

$$
\begin{aligned}
& \lim _{n \rightarrow+\infty} N\left(u_{n}\right)(.)=f\left(., u(.), u^{\prime}(.)\right) \text { in } L^{q}(\Omega) \text { and } \\
& \lim _{n \rightarrow+\infty} \Phi\left(u_{n}^{\prime}(0)\right)=\Phi\left(u^{\prime}(0)\right) \text { in } C(\Omega) .
\end{aligned}
$$

Also from the monotone convergence theorem, we have:

$$
\Xi\left(u_{n}\right)+M u_{n} \rightarrow \Xi(u)+M u \text { in } L^{q}(\Omega) .
$$

Since $M u_{n} \rightarrow M u$ in $C(\Omega)$, it follows that $\Xi\left(u_{n}\right) \rightarrow \Xi(u)$ in $L^{q}(\Omega)$. Using the previous arguments and the dominated convergence theorem, we have:

$$
\lim _{n \rightarrow+\infty} \int_{0}^{t} f\left(t, u_{n}(s), u_{n}^{\prime}(t)\right)+\Xi\left(u_{n}(t)\right) \mathrm{d} t=\int_{0}^{t} f\left(t, u(s), u^{\prime}(t)\right)+\Xi(u(t)) \mathrm{d} t .
$$

It follows that

$$
\begin{aligned}
& \lim _{n \rightarrow+\infty} \Phi\left(u_{n}^{\prime}(0)\right)-\int_{0}^{t} f\left(t, u_{n}(s), u_{n}^{\prime}(t)\right)+\Xi\left(u_{n}(t)\right) \mathrm{d} t \\
& =\Phi\left(u^{\prime}(0)\right)-\int_{0}^{t} f\left(t, u(s), u^{\prime}(t)\right)+\Xi(u(t)) \mathrm{d} t .
\end{aligned}
$$

Since $\Phi$ is an increasing homeomorphism, $\Phi^{-1}$ exists and is continuous. Finally, we have: 


$$
\begin{aligned}
& \lim _{n \rightarrow+\infty} \Phi^{-1} \circ\left(\Phi\left(u_{n}^{\prime}(0)\right)-\int_{0}^{t} f\left(t, u_{n}(s), u_{n}^{\prime}(t)\right)+\Xi\left(u_{n}(t)\right) \mathrm{d} t\right) \\
& =\Phi^{-1} \circ\left(\Phi\left(u^{\prime}(0)\right)-\int_{0}^{t} f\left(t, u(s), u^{\prime}(t)\right)+\Xi(u(t)) \mathrm{d} t\right) \\
& \Leftrightarrow \lim _{n \rightarrow+\infty}\left(K\left(u_{n}\right)\right)^{\prime}=(K(u))^{\prime} \in C(\Omega) .
\end{aligned}
$$

By integration, we obtain: $\lim _{n \rightarrow+\infty} K\left(u_{n}\right)=K(u)$ in $C(\Omega)$. Therefore, $K$ is continuous.

- Let us show that $K$ is completely continuous.

Let $\Pi$ be a bounded set of $C^{1}(\Omega)$. We set $\Delta=\{K(u): u \in \Pi\}$. Since $\Pi$ is bounded, there exist $R>0$ such that:

$$
\|K(u)\|_{\infty}<R+M_{0} T
$$

It follows that:

$$
\|K(u)\|_{C^{1}}<R+\left(M_{0}+1\right) T
$$

Therefore, there exist $R_{1}>0$ such that $K(u) \in \overline{B_{C^{1}}\left(R_{1}\right)}$.

For $u \in \Delta$ and $s_{1}, s_{2} \in \Omega$.

$$
\begin{aligned}
& \left|\Phi(K(u))^{\prime}\left(s_{1}\right)-\Phi(K(u))^{\prime}\left(s_{2}\right)\right| \\
& =\left|\int_{s_{2}}^{s_{1}} N_{f}(u(t))+\hat{\Xi}(u(t)) \mathrm{d} t\right| \leq \sqrt{\left|s_{2}-s_{1}\right|}\left(\left\|\gamma_{r}\right\|_{q}+\|\hat{\Xi}(u)\|_{q}\right)
\end{aligned}
$$

We infer that for all $\epsilon>0$, there exists $\delta>0$ such that

$$
\left|s_{1}-s_{2}\right|<\delta \Rightarrow\left|\Phi(K(u))^{\prime}\left(s_{1}\right)-\Phi(K(u))^{\prime}\left(s_{2}\right)\right|<\epsilon
$$

It suffices to take $\delta=\left(\frac{\epsilon}{\left\|\gamma_{r}\right\|_{q}+\|\hat{\Xi}(u)\|_{q}}\right)^{2}$. Therefore, $\Phi$ being an increasing homeomorphism, for all $\epsilon>0$, it exists $\delta>0$, such that for all $u \in \Pi$, $s_{1}, s_{2} \in \Omega$, if $\left|s_{1}-s_{2}\right|<\delta$, then

$$
\left|(K u)^{\prime}\left(s_{1}\right)-(K u)^{\prime}\left(s_{2}\right)\right|=\left|\Phi^{-1} \circ \Phi\left((K u)^{\prime}\left(s_{1}\right)\right)-\Phi^{-1} \circ \Phi\left((K u)^{\prime}\left(s_{2}\right)\right)\right|<\epsilon .
$$

$\Delta$ is uniformly equicontinuous and $K(\Pi)$ is bounded on $C^{1}(\Omega)$. By Ascoli-Arzela's theorem, $K(\Pi)$ is relatively compact in $C^{1}(\Omega)$. Since $K$ is continuous and $K(\Pi)$ is relatively compact in $C^{1}(\Omega)$ for every bounded subset $\Pi$ of $C^{1}(\Omega), K$ is completely continuous.

\section{Existence Results with Ordered Pair of Lower and Upper Solutions}

We consider the operator $\tau: C^{1}(\Omega) \rightarrow C^{1}(\Omega)$ defined by:

$$
\tau(u)(t)=\max \{\alpha(t), \min \{u(t), \beta(t)\}\}= \begin{cases}\alpha(t) & \text { if } u(t)<\alpha(t) \\ u(t) & \text { if } \alpha(t) \leq u(t) \leq \beta(t) \\ \beta(t) & \text { if } u(t)>\beta(t)\end{cases}
$$


Evidently, $\tau$ is bounded (i.e., maps bounded sets to bounded ones) and is continuous.

Theorem 16. Suppose that there exists a lower solution $\alpha$ and an upper solution $\beta$ such that $\forall t \in[0, T], \alpha(t) \leq \beta(t)$.

Then the problem (1) admits at least one solution $u$, such that:

$$
\alpha(t) \leq u(t) \leq \beta(t), \forall t \in[0, T] .
$$

Moreover, if $\alpha, \beta$ are strict, then

$$
\alpha(t) \leq u(t) \leq \beta(t), \forall t \in[0, T] \text { and } d_{L S}\left[I_{C^{1}}-K, \Pi_{\alpha, \beta}, 0\right]=1 .
$$

where $\Pi_{\alpha, \beta}=\left\{u \in C^{1}(\Omega): \forall t \in[0, T], \alpha(t) \leq u(t) \leq \beta(t),\left\|u^{\prime}\right\|_{\infty}<M_{0}\right\}$

$K$ is the operator associated to the problem (1).

We consider the following auxilary boundary problem:

$$
\left\{\begin{array}{c}
-\left(\Phi\left(u^{\prime}(t)\right)\right)^{\prime}=f_{1}\left(t, u(t), u^{\prime}(t)\right)+\Lambda(t, u(t))+\Xi(u(t))-M \tau(u(t)) \\
+M u(t) \quad \text { a.e on } \Omega=[0, T] \\
u^{\prime}(0) \in B_{1}(u(0)), u^{\prime}(T) \in B_{2}(u(T))
\end{array}\right.
$$

A solution of problem (24) is a function $u \in C^{1}(\Omega)$ such that $\left\|u^{\prime}\right\|_{\infty}<M_{0}$ and satisfied (24).

The problem (24) is equivalent to the fixed point problem $u \in D$ and $u=\bar{K} u$ with $\bar{K}: C^{1}(\Omega) \rightarrow D \subseteq C^{1}(\Omega)$ defined by:

$$
\begin{aligned}
\bar{K} u(t)= & u(0)+\int_{0}^{t} \Phi^{-1} \circ\left(\Phi\left(u^{\prime}(0)\right)-\int_{0}^{y}\left(f_{1}\left(s, u(s), u^{\prime}(s)\right)+\Lambda(s, u(s))\right.\right. \\
& +\Xi(u(s))-M \tau(u(s))+M u(s)) \mathrm{d} s) \mathrm{d} y
\end{aligned}
$$

We have:

$$
\|\bar{K} u\|_{C^{1}}<\max \left\{\|\alpha\|_{\infty},\|\beta\|_{\infty}\right\}+M_{0}(T+1)
$$

Lemma 2. All solution $u$ of (24) is such that $\alpha(t) \leq u(t) \leq \beta(t), \forall t \in[0, T]$.

Proof. Since $\alpha \in C^{1}(\Omega)$ is a lower solution of the problem (1), we have:

$$
\left\{\begin{array}{l}
-\left(\Phi\left(\alpha^{\prime}(t)\right)\right)^{\prime} \leq f\left(t, \alpha(t), \alpha^{\prime}(t)\right)+\Xi(\alpha(t)) \text { a.e on } \Omega=[0, T] \\
\alpha^{\prime}(0) \in B_{1}(\alpha(0))+\mathbb{R}_{+},-\alpha^{\prime}(T) \in B_{2}(\alpha(T))+\mathbb{R}_{+}
\end{array}\right.
$$

Soustraying (25) from (24), we obtain:

$$
\begin{aligned}
& \left(\Phi\left(\alpha^{\prime}(t)\right)\right)^{\prime}-\left(\Phi\left(u^{\prime}(t)\right)\right)^{\prime} \\
& \geq f_{1}\left(t, u(t), u^{\prime}(t)\right)+\Lambda(t, u(t))+\Xi(u(t))-M \tau(u(t))+M w(t) \\
& \quad-f\left(t, \alpha(t), \alpha^{\prime}(t)\right)-\Xi(\alpha(t))
\end{aligned}
$$

We multiply (26) by $(\alpha-u)^{+} \in W^{1, p}((0, T))$ and then integrate on $\Omega$. We obtain:

$$
\int_{0}^{T}\left[\left(\Phi\left(\alpha^{\prime}(t)\right)\right)^{\prime}-\left(\Phi\left(u^{\prime}(t)\right)\right)^{\prime}\right](\alpha-u)^{+}(t) \mathrm{d} t
$$




$$
\begin{aligned}
& \geq \int_{0}^{T}\left[f_{1}\left(t, u(t), u^{\prime}(t)\right)-f\left(t, \alpha(t), \alpha^{\prime}(t)\right)\right](\alpha-u)^{+}(t) \mathrm{d} t \\
& \quad+\int_{0}^{T} \Lambda(t, u(t))(\alpha-u)^{+}(t) \mathrm{d} t \\
& \quad+\int_{0}^{T}[\Xi(u(t))-\Xi(\alpha(t))+M u(t)-M \tau(u(t))](\alpha-u)^{+}(t) \mathrm{d} t .
\end{aligned}
$$

The integration by parts of the left-hand side in inequality, yields:

$$
\begin{aligned}
& \int_{0}^{T}\left[\left(\Phi\left(\alpha^{\prime}(t)\right)\right)^{\prime}-\left(\Phi\left(u^{\prime}(t)\right)\right)^{\prime}\right](\alpha-u)^{+}(t) \mathrm{d} t \\
& =\left(\Phi\left(\alpha^{\prime}(T)\right)-\Phi\left(u^{\prime}(T)\right)\right)(\alpha-u)^{+}(T)-\left(\Phi\left(\alpha^{\prime}(0)\right)-\Phi\left(u^{\prime}(0)\right)\right)(\alpha-u)^{+}(0) \\
& \quad-\int_{0}^{T}\left[\Phi\left(\alpha^{\prime}(t)\right)-\Phi\left(u^{\prime}(t)\right)\right](\alpha-u)^{+}(t) \mathrm{d} t \\
& \geq \int_{0}^{T}\left[f_{1}\left(t, u(t), u^{\prime}(t)\right)-f\left(t, \alpha(t), \alpha^{\prime}(t)\right)\right](\alpha-u)^{+}(t) \mathrm{d} t \\
& \quad+\int_{0}^{T} \Lambda(t, u(t))(\alpha-u)^{+}(t) \mathrm{d} t \\
& \quad+\int_{0}^{T}[\Xi(u(t))-\Xi(\alpha(t))+M u(t)-M \tau(u(t))](\alpha-u)^{+}(t) \mathrm{d} t .
\end{aligned}
$$

We set

$$
\left[(\alpha-u)^{+}\right](t)= \begin{cases}(\alpha(t)-u(t)) & \text { if } \alpha(t)>u(t) \\ 0 & \text { if } \alpha(t) \leq u(t)\end{cases}
$$

Also, from the boundary conditions in (24) and (25), we have:

$$
-u^{\prime}(T) \in B_{1}(u(T)) \text { and }-\alpha^{\prime}(T) \in B_{1}(\alpha(T))+e_{T} \text { with } e_{T} \geq 0 .
$$

If $\alpha(T) \geq u(T)$, then from the monotony of $B_{2}$ (See hypothesis $\left(H_{B}\right)$ ), we have:

$$
\alpha^{\prime}(T) \leq u^{\prime}(T) \text {. Whence } \Phi\left(\alpha^{\prime}(T)\right) \leq \Phi\left(u^{\prime}(T)\right) .
$$

So, it follows that

$$
\left(\Phi\left(\alpha^{\prime}(T)\right)-\Phi\left(u^{\prime}(T)\right)\right)(\alpha-u)(T) \leq 0 .
$$

In a similar way, using the boundary conditions $u^{\prime}(0) \in B_{1}(u(0))$ and $\alpha^{\prime}(0) \in B_{1}(\alpha(0))+e_{0}$ with $e_{0} \geq 0$, if $\alpha(0) \geq u(0)$, we have:

$$
\alpha^{\prime}(0) \geq u^{\prime}(0) \text {. We infer that } \Phi\left(\alpha^{\prime}(0)\right) \geq \Phi\left(u^{\prime}(0)\right) \text {. }
$$

It follows that

$$
\left(\Phi\left(\alpha^{\prime}(0)\right)-\Phi\left(u^{\prime}(0)\right)\right)(\alpha-u)(0) \geq 0 .
$$

Also, since $\Phi$ is an increasing homeomorphism, we have:

$$
\begin{aligned}
& \int_{0}^{T}\left(\Phi\left(\alpha^{\prime}(t)\right)-\Phi\left(u^{\prime}(t)\right)\right)(\alpha-u)^{+}(t) \mathrm{d} t \\
& =\int_{\alpha>u}\left(\Phi\left(\alpha^{\prime}(t)\right)-\Phi\left(u^{\prime}(t)\right)\right)(\alpha-u)(t) \mathrm{d} t \geq 0
\end{aligned}
$$

where $\{\alpha>u\}=\{t \in[0, T]: \alpha(t)>u(t)\}$.

Using the inequalities (30), (31) and (32) in the first member of (27), we obtain:

$$
\int_{0}^{T}\left[\left(\Phi\left(u^{\prime}(t)\right)\right)^{\prime}-\left(\Phi\left(\alpha^{\prime}(t)\right)\right)^{\prime}\right](\alpha-u)^{+}(t) \mathrm{d} t \leq 0 .
$$


Furthermore:

$$
\begin{aligned}
& f_{1}\left(t, u(t), u^{\prime}(t)\right)-f\left(t, \alpha(t), \alpha^{\prime}(t)\right) \\
& =f\left(t, \alpha(t), \alpha^{\prime}(t)\right)-f\left(t, \alpha(t), \alpha^{\prime}(t)\right)=0 \text { a.e on }\{\alpha>u\} \\
& \Rightarrow \int_{0}^{T}\left(f_{1}\left(t, u(t), u^{\prime}(t)\right)-f\left(t, \alpha(t), \alpha^{\prime}(t)\right)\right)(\alpha-u)^{+}(t) \mathrm{d} t=0 .
\end{aligned}
$$

Also from the definiton of the penalty map $\Lambda$, if $|\{\alpha>u\}|>0$ (By $|\cdot|$, we denote the Lebesgue mesure in $\mathbb{R}$ ), then:

$$
\int_{0}^{T} \Lambda(t, u(t))(\alpha-u)^{+}(t) \mathrm{d} t=\int_{\alpha>u}(\Phi(\alpha(t))-\Phi(u(t)))(\alpha-u)^{+}(t) \mathrm{d} t>0 .
$$

Finally, by virtue of hypothesis $\left(H_{\Xi}\right)$ and since $w \in U$, we see that:

$$
\begin{aligned}
& \int_{0}^{T}[\Xi(u(t))-\Xi(\alpha(t))-M \tau(u(t))-M u(t)](\alpha-u)^{+}(t) \mathrm{d} t \\
& =\int_{\alpha>u}[\Xi(u(t))-\Xi(\alpha(t))-M \alpha(t)+M u(t)](\alpha-u)^{+}(t) \mathrm{d} t \geq 0 .
\end{aligned}
$$

Using the inequalities, (34), (35) and (36) in the second member of (27), we infer that:

$$
\begin{aligned}
& \int_{0}^{T}\left[f_{1}\left(t, u(t), u^{\prime}(t)\right)-f\left(t, \alpha(t), \alpha^{\prime}(t)\right)\right](\alpha-u)^{+} \mathrm{d} t \\
& +\int_{0}^{T} \Lambda(t, u(t))(\alpha-u)^{+}(t) \mathrm{d} t \\
& +\int_{0}^{T}[\Xi(u(t))-\Xi(\alpha(t))-M \tau(u(t))+M u(t)](\alpha-u)^{+}(t) \mathrm{d} t>0 .
\end{aligned}
$$

We consider (27) and using (33) and (34), we have a contradiction when $|\{\alpha>u\}|>0$. Therefore, for all $t \in \Omega, \alpha(t) \leq u(t)$. In a similar fashion we show that $u(t) \leq \beta(t)$ for all $t \in \Omega$. Thus $u \in U$.

Proof. theorem 16: As in the proof of the proposition 15, we can show the complete continuity of the operator $\bar{K}$. Moreover, $\bar{K}\left(C^{1}\right) \subset B_{C^{1}}(R)$ for all $R>\max \left\{\|\alpha\|_{\infty},\|\beta\|_{\infty}\right\}+M_{0}(T+1)$. Therefore, by Leray-Schauder's theorem, we can say that the operator $\bar{K}$ has a fixed point $u$ in the open ball $B_{C^{1}}(R)$ which is solution of problem (24). It follows, by the lemma 2, that $u$ is also solution of problem (1).

We assume that $\alpha$ is a strict lower solution and $\beta$ is a strict upper solution of (1). Let

$$
R>\max \left\{\|\alpha\|_{\infty},\|\beta\|_{\infty}\right\}+M_{0}(T+1)
$$

be quite a few such that

$$
\bar{K} u \neq u \text { for some } u \in \partial B_{C^{1}}(R) .
$$

Because $\bar{K}$ is completely continuous, we can compute the degree of $I d_{C^{1}}-\bar{K}$. The function $H$ defined by $H(t, u)=t \bar{K}(u)$ is compact on $[0,1] \times B_{C^{1}}(R)$. We assume that there exist $t \in[0,1]$ and $v \in \partial B_{C^{1}}(R)$ such that $v-H(v)=0$, then $v=t \bar{K}(v)$. But $\|v\|_{C^{1}}=R$, so $t\|\bar{K}(v)\|_{C^{1}}=R$ which contradict the fact that $\|\bar{K}(v)\|_{C^{1}}<R$. We can apply the homotopic invariance degre property of Leray-Schauder to obtain:

$$
d_{L S}\left[I d_{C^{1}}-\bar{K}, B_{C^{1}}(R), 0\right]=d_{L S}\left[I d_{C^{1}}-K, B_{C^{1}}(R), 0\right]=1 .
$$


Indeed, let us recall the following set:

$$
\Pi_{\alpha, \beta}=\left\{u \in C^{1}: \forall t \in[0, T], \alpha(t) \leq u(t) \leq \beta(t),\left\|u^{\prime}\right\|_{\infty}<M_{0}\right\} .
$$

By definitions of strict lower and strict upper solutions, $\alpha$ and $\beta$ cannot be solution of problem (24). Therefore, (24) has not solution on the boundary of $\Pi_{\alpha, \beta}$. By using the additivity and excision properties of Leray-Schauder degree, we obtain:

$$
d_{L S}\left[I d_{C^{1}}-\bar{K}, \Pi_{\alpha, \beta}, 0\right]=d_{L S}\left[I d_{C^{1}}-\bar{K}, B_{C^{1}}(R), 0\right]=1 .
$$

Furthermore, since $K$ is completely continuous operator associate to (1). That equates to $\bar{K}$ on $\Pi_{\alpha, \beta}$, we have

$$
d_{L S}\left[I d_{C^{1}}-K, B_{C^{1}}(R), 0\right]=1 .
$$

\section{Existence Results with Non Ordered Lower and Upper Solutions}

Theorem 17. We assume that there exists a lower solution $\alpha$ and an upper solution $\beta$ of $(1)$ such that

$$
\exists \bar{t} \in[0, T] \text { such that } \alpha(\bar{t})>\beta(\bar{t}) .
$$

Then the problem (1) admits at least one solution $u$, such that:

$$
\min \left\{\alpha_{u}, \beta_{u}\right\} \leq u\left(t_{u}\right) \leq \max \left\{\alpha_{u}, \beta_{u}\right\} \text { for some } t_{u} \in[0, T]
$$

and

$$
\|u\|_{\infty} \leq \max \left\{\|\alpha\|_{\infty},\|\beta\|_{\infty}\right\}+M_{0} T .
$$

Proof. We set $\lambda=\max \left\{\|\alpha\|_{\infty},\|\beta\|_{\infty}\right\}+M_{0} T$.

We consider the functions $f^{*}:[0, T] \times \mathbb{R}^{2} \rightarrow \mathbb{R}, \Xi^{*}: \mathbb{R} \rightarrow \mathbb{R}$, and for $i=1,2$, the multifunctions $B_{i}^{*}: \mathbb{R} \rightarrow P(\mathbb{R})$ are defined respectively by:

$$
\begin{gathered}
f^{*}(t, u, v)= \begin{cases}2 & \text { if } u>\lambda+1 \\
(1+\lambda-u) f(t, u, v)+2(u-\lambda) & \text { if } \lambda<u \leq \lambda+1 \\
f(t, u, v) & \text { if }-\lambda \leq u \leq \lambda \\
(1+\lambda+u) f(t, u, v)+2(u+\lambda) & \text { if }-\lambda-1 \leq u<-\lambda \\
-2 & \text { if } u<-\lambda-1\end{cases} \\
\Xi^{*}(u)= \begin{cases}1 & \text { if } u>\lambda+1 \\
(1+\lambda-u) \Xi(u)+(u-\lambda) & \text { if } \lambda<u \leq \lambda+1 \\
\Xi(u) & \text { if }-\lambda \leq u \leq \lambda \\
(1+\lambda+u) \Xi(u)+(u+\lambda) & \text { if }-\lambda-1 \leq u<-\lambda \\
-1 & \text { if } u<-\lambda-1\end{cases} \\
B_{1}^{*}(u)= \begin{cases}p_{1}(+)+3 & \text { if } u>\lambda+1 \\
p_{1}(+)+3(u-\lambda) & \text { if } \lambda<u \leq \lambda+1 \\
B_{1}(u) & \text { if }-\lambda \leq u \leq \lambda \\
p_{1}(-)+3(u+\lambda) & \text { if }-\lambda-1 \leq u<-\lambda \\
p_{1}(-)-3 & \text { if } u<-\lambda-1\end{cases}
\end{gathered}
$$




$$
B_{2}^{*}(u)= \begin{cases}p_{2}(-)-3 & \text { if } u>\lambda+1 \\ p_{2}(-)-3(u-\lambda) & \text { if } \lambda<u \leq \lambda+1 \\ B_{2}(u) & \text { if }-\lambda \leq u \leq \lambda \\ p_{2}(+)-3(u+\lambda) & \text { if }-\lambda-1 \leq u<-\lambda \\ p_{2}(+)+3 & \text { if } u<-\lambda-1\end{cases}
$$

where, for $i=1,2, p_{i}(+)$ and $p_{i}(-)$ are defined as in remark $10, f^{*}$ is $L^{p}$-Caratheodory and $B_{1}^{*}$ and $B_{2}^{*}$ are maximal monotone operators. We consider the following modified problem:

$$
\left\{\begin{array}{l}
-\left(\Phi\left(u^{\prime}(t)\right)\right)^{\prime}=f^{*}\left(t, u(t), u^{\prime}(t)\right)+\Xi^{*}(u(t)) \text { a.e on }[0, T] \\
u^{\prime}(0) \in B_{1}^{*}(u(0)),-u^{\prime}(T) \in B_{2}^{*}(u(T)) .
\end{array}\right.
$$

We can verify that $\alpha$ is a lower solution and $\beta$ is upper solution of the problem (41). Let $\tilde{\beta}: \mathbb{R} \rightarrow \mathbb{R}$ be defined by $\tilde{\beta}(t)=\lambda+2, \forall t \in \mathbb{R}$. We have:

$$
\begin{gathered}
\left(\Phi\left(\tilde{\beta}^{\prime}(t)\right)\right)^{\prime}=0<3=f^{*}\left(t, u(t), u^{\prime}(t)\right)+\Xi^{*}(u(t)) \text { a.e on }[0, T], \\
\tilde{\beta}^{\prime}(0)=0<p_{1}(+)+3 \text { and }-\tilde{\beta}^{\prime}(T)=0<p_{2}(+)+3 .
\end{gathered}
$$

So we can find $e_{0}, e_{T} \geq 0$ such that

$$
\tilde{\beta}^{\prime}(0) \in B_{2}^{*}(\tilde{\beta}(0))-e_{0} \text { and }-\tilde{\beta}^{\prime}(T) \in B_{2}^{*}\left(\tilde{\beta}^{\prime}(T)\right)-e_{T}
$$

Therefore, $\tilde{\beta}$ is an upper solution of (41).

The function $\tilde{\alpha}: \mathbb{R} \rightarrow \mathbb{R}$ defined by $\tilde{\alpha}(t)=-\lambda-2, \forall t \in \mathbb{R}$, verifies:

$$
\begin{gathered}
\left(\Phi\left(\tilde{\alpha}^{\prime}(t)\right)\right)=0>-3=f^{*}\left(t, u(t), u^{\prime}(t)\right)+\Xi^{*}(u(t)) \text { a.e on }[0, T], \\
\tilde{\alpha}^{\prime}(0)=0>p_{1}(-)-3,-\tilde{\alpha}^{\prime}(T)=0>-p_{2}(-)-3 .
\end{gathered}
$$

So we can find $l_{0}, l_{T} \geq 0$ such that

$$
\tilde{\alpha}^{\prime}(0) \in B_{2}^{*}(\tilde{\alpha}(0))+l_{0} \text { and }-\tilde{\alpha}^{\prime}(T) \in B_{2}^{*}(\tilde{\alpha}(T))+l_{T}
$$

Therefore, $\tilde{\alpha}$ is a lower solution of (41). Furthermore,

$$
\forall t \in[0, T], \tilde{\alpha} \leq \min \{\alpha, \beta\} \leq \max \{\alpha, \beta\} \leq \tilde{\beta} .
$$

Let us introduce the sets

$$
\begin{aligned}
& \Pi_{\tilde{\alpha}, \beta}=\left\{u \in C^{1}: \forall t \in[0, T], \tilde{\alpha}(t)<u(t)<\beta(t),\left\|u^{\prime}\right\|_{\infty}<M_{0}\right\}, \\
& \Pi_{\alpha, \tilde{\beta}}=\left\{u \in C^{1}: \forall t \in[0, T], \alpha(t)<u(t)<\tilde{\beta}(t),\left\|u^{\prime}\right\|_{\infty}<M_{0}\right\},
\end{aligned}
$$

and

$$
\Pi_{\tilde{\alpha}, \tilde{\beta}}=\left\{u \in C^{1}: \forall t \in[0, T], \tilde{\alpha}(t)<u(t)<\tilde{\beta}(t),\left\|u^{\prime}\right\|_{\infty}<M_{0}\right\} .
$$

By using the definition (39), we obtain:

$$
\Pi_{\tilde{\alpha}, \beta} \cap \Pi_{\alpha, \tilde{\beta}}=\varnothing .
$$

Also we have:

$$
\Pi_{\tilde{\alpha}, \beta} \cup \Pi_{\alpha, \tilde{\beta}} \subset \Pi_{\tilde{\alpha}, \tilde{\beta}} \cdot
$$


Let us consider:

$$
\Pi=\Pi_{\tilde{\alpha}, \tilde{\beta}} \backslash\left(\bar{\Pi}_{\tilde{\alpha}, \beta} \cup \bar{\Pi}_{\alpha, \tilde{\beta}}\right) .
$$

Then

$$
\Pi=\left\{u \in \Pi_{\tilde{\alpha}, \tilde{\beta}}: \exists\left(t_{1}, t_{2}\right) \in[0, T]^{2} \text { such that }\left(\left(t_{1}\right)<u\left(t_{1}\right) \text { and } u\left(t_{2}\right)<\alpha\left(t_{2}\right)\right\}\right.
$$

and

$$
\partial \Pi_{\tilde{\alpha}, \tilde{\beta}}=\partial \Pi_{\alpha, \tilde{\beta}} \cup \partial \Pi_{\alpha, \tilde{\beta}} \cup \partial \Pi
$$

Since all constant function between $\beta(\tilde{t})$ and $\alpha(\tilde{t})$ is into $\Pi, \Pi$ is nonempty.

Let $K^{*}$ be the fixed point operator associated to problem (41) given in the proposition 15. We consider $u \in \Pi$ such that $K^{*}(u)=u$ and $\|u\|_{\infty}=\lambda+2$. There exists $t_{0} \in[0, T]$ such that

$$
u\left(t_{0}\right)=\max _{[0, T]} u=\lambda+2 \text { or } u\left(t_{0}\right)=\min _{[0, T]} u=-\lambda-2 .
$$

Let us consider the case $u\left(t_{0}\right)=\max _{[0, T]} u=\lambda+2$.

If $t_{0} \in[0, T]$, then $u^{\prime}\left(t_{0}\right)=0$ and there exists $\epsilon>0$ such that $u(t)>\lambda+1$ for all $t \in\left[t_{0}, t_{0}+\epsilon\right]$. Moreover $\left(\Phi\left(u^{\prime}(t)\right)\right)^{\prime}=3$. Whence,

$$
\Phi\left(u^{\prime}(t)\right)=\int_{t_{0}}^{t}\left(\Phi\left(u^{\prime}(t)\right)\right)^{\prime} \mathrm{d} t>0,
$$

for all $t \in\left[t_{0}, t_{0}+\epsilon\right]$. It follows that $u$ is increasing on $\left[t_{0}, t_{0}+\epsilon\right]$. That contradicts the existence of $t_{0}$.

If $t=0, u^{\prime}(0)=0$ and we obtain the contradiction $0=p_{1}(+)+3$.

If $t_{0}=T, u^{\prime}(T)=0$ and we obtain the contradiction $0=p_{2}(-)-3$.

In the similar fashion, we obtain contradiction with the case $u\left(t_{0}\right)=\min _{[0, T]} u=-\lambda-2$. Therefore

$$
\left[u \in \partial \Pi, K^{*}(u)=u\right] \Rightarrow\|u\|_{\infty}<\lambda+2 .
$$

Let $u \in \partial \Pi$ such that $K^{*} u=u$. It becomes from (42) that $\|u\|_{\infty}<\lambda+2$, $\left\|u^{\prime}\right\|_{\infty}<M_{0}$, and $u \in \partial \Pi_{\tilde{\alpha}, \beta} \cup \partial \Pi_{\alpha, \tilde{\beta}}$. It follows, there exists $t_{0} \in[0, T]$ such that $u\left(t_{0}\right)=\alpha\left(t_{0}\right)$ and $u\left(t_{0}\right)=\beta\left(t_{0}\right)$, that implies

$$
\left|u\left(t_{0}\right)\right|<\max \left\{\|\alpha\|_{\infty},\|\beta\|_{\infty}\right\}
$$

Then,

$$
|u(t)| \leq\left|u\left(t_{0}\right)\right|+\int_{0}^{T}\left|u^{\prime}(t)\right| \mathrm{d} t<\lambda, \forall t \in[0, T]
$$

So,

$$
\left[u \in \partial \Pi, K^{*}(u)=u \Rightarrow\|u\|_{\infty}<\lambda\right]
$$

We have two cases:

- $1^{\text {rst }}$ case: We assume that there exists $u \in \partial \Pi$ such that $K^{*}(u)=u$. From (43), we infer that $\|u\|_{\infty}<\lambda$, that implies that $u$ is a solution of (1), and (39) and (40) are satisfied. Then, there exists $\varsigma \in[0, T]$ such that $u(\varsigma)=\alpha(\varsigma)$ or $u(\varsigma)=\beta(\varsigma)$, 
- $2^{\text {nd }}$ case: We assume that $K^{*}(u) \neq u$ for all $u \in \partial \Pi$. Then, as in the proof of theorem 16, we have:

$$
\begin{aligned}
& d_{L S}\left(I d_{C^{1}}-K^{*}, \Pi_{\tilde{\alpha}, \tilde{\beta}}, 0\right) \\
& =d_{L S}\left(I d_{C^{1}}-K^{*}, \Pi_{\tilde{\alpha}, \beta}, 0\right)=d_{L S}\left(I_{C^{1}}-K^{*}, \Pi_{\alpha, \tilde{\beta}}, 0\right)=1
\end{aligned}
$$

Theorem 18. If there exists a lower solution $\alpha$ and an upper solution $\beta$ of the problem (1), then the problem (1) admits at least one solution u such that.

$$
\|u\|_{\infty} \leq \max \left\{\|\alpha\|_{\infty},\|\beta\|_{\infty}\right\}+M_{0} T .
$$

Proof. If $\forall t \in[0, T], \alpha(t) \leq \beta(t)$, by theorem 16 , the problem (1) admits at least one solution such that:

$$
\alpha(t) \leq u(t) \leq \beta(t), \forall t \in[0, T]
$$

Moreover, (44) holds.

If

$$
\exists \tilde{t} \in[0, T] \text { such that } \alpha(\tilde{t})>\beta(\tilde{t})
$$

By the theorem 17, the problem (1) admits at least one solution, such that:

$$
\|u\|_{\infty} \leq \max \left\{\|\alpha\|_{\infty},\|\beta\|_{\infty}\right\}+M_{0} T .
$$

\section{Multiplicity Results}

Theorem 19. We assume that there exists $\alpha$ a lower solution and $\tilde{\alpha}$ a strict lower solution of problem (1), $\beta$ an upper solution and $\tilde{\beta}$ a strict upper solution of problem (1) such that:

$$
\forall t \in[0, T], \alpha(t) \leq \beta(t) \leq \tilde{\alpha}(t) \leq \tilde{\beta}(t) .
$$

Then the problem (1) admits at least three solutions $u, v$ and $w$ such that:

$$
\begin{gathered}
\alpha(t) \leq u(t) \leq \beta(t), t \in[0, T] ; \tilde{\alpha}(t) \leq v(t) \leq \tilde{\beta}(t), t \in[0, T] ; \\
\beta\left(t_{w}\right) \leq u\left(t_{w}\right) \leq \tilde{\alpha}\left(t_{w}\right) \text { for some } t_{w} \in[0, T] .
\end{gathered}
$$

Proof. By using the theorem 16 and the fact that $\beta$ and $\tilde{\beta}$ are strict, we can say that the problem (1) admits at least one solution $u$, such that:

$$
\beta(t) \leq u(t) \leq \tilde{\beta}(t)
$$

By using the theorem 16, the problem (1) admits at least one solution $w$ such that:

$$
\begin{aligned}
\beta\left(t_{w}\right) & =\min \left\{\tilde{\alpha}, \beta\left(t_{w}\right)\right\} \leq w\left(t_{w}\right) \leq \max \left\{\tilde{\alpha}, \beta\left(t_{w}\right)\right\} \\
& =\tilde{\alpha}(t) \text { for some } t_{w} \in[0, T] .
\end{aligned}
$$

(46) and (47) yield $u \neq w$ and $w \neq v$.

In the following theorem, we show existence of at least two solutions of the problem (1).

Theorem 20. We assume that there exists $\alpha$ and $\tilde{\alpha}$ are two lower solutions of the problem (1), $\beta$ is a strict upper solution of problem (1) such that. 


$$
\forall t \in[0, T], \alpha(t) \leq \beta(t) \leq \tilde{\beta}(t) .
$$

Then the problem (1) admits at least two solutions $u$ and $w$ such that:

$$
\alpha(t) \leq u(t) \leq \beta(t), t \in[0, T]
$$

and

$$
\exists t \in[0, T] \text { such that } \beta\left(t_{w}\right) \leq u\left(t_{w}\right) \leq \tilde{\alpha}\left(t_{w}\right) .
$$

Proof. By using the theorem 16 and the fact that $\beta$ is strict, we can say that the problem (1) admits at least one solution $u$, such that:

$$
\alpha(t) \leq u(t) \leq \beta(t) .
$$

By using the theorem 17, the problem (1) admits at least one solution $w$ such that:

$$
\begin{aligned}
\beta\left(t_{w}\right) & =\min \left\{\tilde{\alpha}\left(t_{w}\right) \beta\left(t_{w}\right)\right\} \leq w\left(t_{w}\right) \leq \max \left\{\tilde{\alpha}\left(t_{w}\right), \beta\left(t_{w}\right)\right\} \\
& =\tilde{\beta}(t) \text { for some } t_{w} \in[0, T] .
\end{aligned}
$$

(49) and (50) yield $u \neq w$.

Theorem 21. We assume that there exists $\alpha$ a strict lower solutions of the problem (1) and two upper solutions $\beta$ and $\tilde{\beta}$ of the problem(1) such that:

$$
\forall t \in[0, T], \beta(t) \leq \tilde{\beta}(t) .
$$

Then the problem (1) admits at least two solutions $u$ and $w$ such that:

$$
\alpha(t) \leq u(t) \leq \tilde{\alpha}(t), t \in[0, T]
$$

and

$$
\exists t_{w} \in[0, T] \text { such that } \beta\left(t_{w}\right) \leq u\left(t_{w}\right) \leq \alpha\left(t_{w}\right) .
$$

Proof. The proof is similar to those of theorem 18.

\section{Example and Periodic Problem}

\subsection{Example}

Let us consider the following problem:

$$
\left\{\begin{array}{l}
-\left(\left.\frac{\sqrt{1+\left(1+\left|u^{\prime}(t)\right|^{p-1}\right)^{2}}}{1+\left|u^{\prime}(t)\right|^{p-1}} u^{\prime}(t)\right|^{p-2} u^{\prime}(t)\right)=f\left(t, u(t), u^{\prime}(t)\right)+\Xi(u(t)) \text { a.e on } \Omega=[0, T] \\
u^{\prime}(0) \in B_{1}(u(0)),-u^{\prime}(T) \in B_{2}(u(T))
\end{array}\right.
$$

where $f, \Xi, B_{1}$ and $B_{2}$ are defined as in problem (1). Here,

$$
\Phi(z)=\frac{\sqrt{1+\left(1+|z|^{p-1}\right)^{2}}}{1+|z|^{p-1}}|z|^{p-2} z,
$$

for all $z \in \mathbb{R}$ and by the remark 8 , it satisfies hypothesis $\left(H_{\Phi}\right)$. Therefore, theorem 16 and theorem 17 are true for the problem (52). Moreover, by [8] (see example 5.2.25 page 404), this problem unifies classical problems of Dirichlet, 
Neumann and Sturm-Liouville and go beyong them.

\subsection{Periodic Problem}

Let us consider the following periodic problem:

$$
\left\{\begin{array}{l}
-\left(\Phi\left(u^{\prime}(t)\right)\right)^{\prime}=f\left(t, u(t), u^{\prime}(t)\right)+\Xi(u(t)) \text { a.e on } \Omega=[0, T] \\
u(0)=u(T), u^{\prime}(0)=u^{\prime}(T) .
\end{array}\right.
$$

Remark 22. The theorems 16 and 17 stay true for this problem (see 8 remark 5.2.26 page 404 and also [5] section 6 page 23).

\section{Conclusion}

In this article, by combining lower and upper solutions method, theory of maximal monotone operators and theory of topological degree, we establish existence and multiplicity results for second-order problems with multivalued boundary conditions. We give an example but more examples and applications can be given. In perspective, we will study the same problems under general multivalued boundary conditions. Also, the same problem can be considered for a singular $\Phi$-Laplacian operator.

\section{Conflicts of Interest}

The authors declare no conflicts of interest regarding the publication of this paper.

\section{References}

[1] Perron, O. (1923) Eine neue Behandlung der Randwertaufgabe für $\Delta u=0$. Mathematische Zeitschrift, 18, 42-54. https://doi.org/10.1007/BF01192395

[2] Nagumo (1937) Uber die Differentialgleichung $y^{\prime \prime}=f\left(t, y, y^{\prime}\right)$. Proceedings of the Physico-Mathematical Society of Japan, 19, 861-866.

[3] Goli, C.E. and Adje, A. (2017) Existence of Solutions of Some Nonlinear $\Phi$-Laplacian Equations with Neumann-Steklov Nonlinear Boundary Conditions. African Diaspora Journal of Mathematics, 20, 16-38.

[4] Khattabi, N.E., Frigon, M. and Ayyadi, N. (2013) Multiple Solutions of Boundary Value Problems with $\varphi$-Laplacian Operators and under a Wintner-Nagumo Growth Condition. Boundary Value Problems, 2013, 236. https://doi.org/10.1186/1687-2770-2013-236

[5] Bader, R. and Papageorgioua, N. (2001) Nonlinear Multivalued Boundary Value Problems Discussiones Mathematicae Differential Inclusions. Control and Optimization, 21, 127-148. https://doi.org/10.7151/dmdico.1020

[6] Kandilakis, D.A. and Papageorgiou, N. (1996) Existence Theorems for Nonlinear Boundary Value Problems for Second Order Differential Inclusion. Journal of Differential Equations, 132, 107-125. https://doi.org/10.1006/jdeq.1996.0173

[7] Halidias, N. and Papageorgiou, N. (1998) Existence and Relaxation Results for Nonlinear Second Order Multivalued Boundary Value Problems in $\mathrm{R}^{\mathrm{N}}$. Journal of Differential Equations, 147, 123-154. https://doi.org/10.1006/jdeq.1998.3439 
[8] Papageorgiou, N. and Kyritsi, S.T. (2009) Handbook of Applied Analysis. Springer Science + Business Media, Berlin, Vol. 19, 390-404.

[9] Staicub, V. and Papageorgiou, N. (2007) The Method of Upper Lower Solutions for Nonlinear Second Order Differential Inclusions. Nonlinear Analysis, 67, 708-726. https://doi.org/10.1016/j.na.2006.06.023

[10] Hu, S. and Papageorgiou, N. (1997) Handbook of Multivalued Analysis. Volume I: Theory. Kluwer, Dordrecht. https://doi.org/10.1007/978-1-4615-6359-4

[11] Zeidler, E. (1990) Nonlinear Functional Analysis and Its Applications II. Springer-Verlag, New York. https://doi.org/10.1007/978-1-4612-0981-2

[12] Deimling, K. (1985) Nonlinear Functional Analysis. Springer, Berlin. https://doi.org/10.1007/978-3-662-00547-7

[13] Kyritsi, S., Matzakos, N. and Papageorgiou, N. (2002) Periodic Problems for Strongly Nonlinear Second Order Differential Inclusions. Journal of Differential Equations, 183, 279-302. https://doi.org/10.1006/jdeq.2001.4110

[14] Manasevich, R. and Mawhin, J. (1998) Periodic Solutions for Nonlinear Systems with p-Laplacianlike Operators. Differential Equations, 145, 367-393. https://doi.org/10.1006/jdeq.1998.3425

[15] Brezis, H. (1973) Opérateurs Maximaux Monotones. North-Holland, Amsterdam. 